\title{
Über die Konstitution der Ferrisalze der unterphosphorigen Săure.
}

\author{
Von R.F. WEINLAND und W. HIEBER.
}

Im letzten Jahrzehnt wurde, wie bekannt, die Konstitution der Ferrisalze der nichtsubstituierten Monocarbonsäuren aufgeklärt. ${ }^{1}$ ) Sie enthalten alle - seien sie wasserlöslich wie die Ferriacetate oder wasserunlöslich wie die Ferribenzoate - ein eigentümlich zusammengesetztes, mehrkerniges Kation, das aus 3 Atomen Eisen und 6 Säureresten besteht und von dem man mit beliebigen Säuren Salze darstellen kann, z. B. Salze des Hexaacetato(benzoato)triferri-kations:

$$
\begin{aligned}
& \left.\left[\mathrm{Fe}_{3} \underset{(\mathrm{OH})_{2}}{\left(\mathrm{CH}_{3} \mathrm{COO}\right)_{8}}\right] \mathrm{NO}_{3}+4 \mathrm{H}_{2} \mathrm{O}^{2}\right) \text {, Nitrat; } \\
& {\left[\mathrm{Fe}_{3} \underset{(\mathrm{OH})_{2}}{\left(\mathrm{CH}_{3} \mathrm{COO}\right)_{6}}\right]_{1 / 2} \mathrm{Pt}(\mathrm{Sn}) \mathrm{Cl}_{6}+5 \mathrm{H}_{2} \mathrm{O} \text {, Chloroplatinat(-stannat); }} \\
& {\left[\mathrm{Fe}_{3}{ }^{\left(\mathrm{CH}{ }_{3} \mathrm{COO}\right)_{6}}\right] \mathrm{CH}_{3} \mathrm{COO}+\mathrm{H}_{2} \mathrm{O} \text {, Monoacetat; }}
\end{aligned}
$$

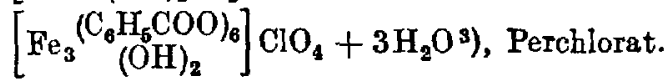

Die Ferriacetate sind demnach Acetate jenes Kations. Wir bemerken, daß die meisten dieser Salze basisch sind, daher die Hydroxylgruppen in den Formeln.

Ein solches komplexes Kation war bisher nur bei organischen Säuren beobachtet worden. Wir haben nun gefunden, daß auch den Ferrih y po phos phi ten ein derartiges $\mathrm{Ka}$ tion zugrunde liegt, das aus 3 Atomen Fisen und 6 Unterphosphorigsäureresten besteht:

$$
\left[\mathrm{Fe}_{3}\left(\mathrm{H}_{2} \mathrm{PO}_{2}\right)_{8}\right] \text {. }
$$

Bis jetzt war nur ein Ferrihypophosphit bekannt, das von H. Rose durch Einwirkung von verdünter unterphosphoriger Säure auf frisch gefälltes Ferrihydroxyd dargestellt' worden war. Es bildet ein sandiges, weißliches Pulver mit einem ganz schwachen Stich ins Rötliche und ist durch große Beständigkeit ausgezeichnet (s. im Folgenden). Rein stöchiometrisch kommt ihm die Zusammensetzung eines normalen Salzes zu:

$$
\mathrm{Fe}\left(\mathrm{H}_{2} \mathrm{PO}_{2}\right)_{3} \text {. }
$$

1) Zusammenfassende Abhandlung, R. WrRNLAND und FR. PASCHEN, Z. anorg. u. allg. Chem. 82 (1915), 81.

2) R. Whancard und E. Gussmans, Ber. deutsch. chem. Ges. 42 (1909), 3881.

of R. WhanLawd und A. HrRz, Ber. 45 (1912), 2662. 
Es ist an sioh schon eine interessante Tatsache, da $B$ das dreiwertige Fisen mit dieser stark reduzierenden Säure eine so beständige Verbindung bildet.

Nach der angeführten Methode von Rose Ferrihypophosphit in größerer Menge darzustellen, beansprucht jedoch manchmal ziemlich lange Zeit, auch sind die Ausgangsprodukte selbst in der hierzu erforderlichen Reinheit und Konzentration nicht so leicht zugänglich. Wir gingen daher für unsere Untersuchung von einem Ferrihypophosphit aus, das man durch Versetzen von Natriumhypophosphitlösung mit Ferrichlorid als pulverförmigen weißlichen Niederschlag bekommt, und das im wesentlichen ein basisches Salz der Zusammensetzung

vorstellt.

$$
\mathrm{Fe}_{3}\left(\mathrm{H}_{2} \mathrm{PO}_{2}\right)_{8}(\mathrm{OH})
$$

Aus diesem Ferrihypophosphit erhielten wir durch Einwirkung anorganischer Säuren eine Reihe von zum Teil ausgezeichnet kristallisierten Verbindungen, welche gleichzeitig dreiwertiges Eisen, unterphosphorige Säure und die betreffende anorganische Säure enthalten. Sie entstehen teils durch Umwandlung des Ferrihypophosphits in der einwirkenden Säure, teils kristallisieren sie aus der Lösung des Ferrihypophosphits aus der wäßrigen Säure aus, endlich erbält man sie aus der Lösung in konzentrierteren Säuren durch Zusatz von Wasser oder Alkohol (Genaueres s. exper. Teil).

Es sind die folgenden:

$\left.\begin{array}{l}\text { 1. } \mathrm{Fe}_{3}\left(\mathrm{H}_{2} \mathrm{PO}_{2}\right)_{7} \mathrm{SO}_{4} \cdot 4 \mathrm{H}_{2} \mathrm{O} \\ \text { 2. } \mathrm{Fe}_{3}\left(\mathrm{H}_{2} \mathrm{PO}_{2}\right)_{8} \mathrm{SO}_{4 / 2}+4 \mathrm{H}_{2} \mathrm{O}\end{array}\right\}$ kristallisiert, blaßrot.

3. $\mathrm{Fe}_{3}\left(\mathrm{H}_{2} \mathrm{PO}_{2}\right)_{6}\left(\mathrm{SO}_{4} \mathrm{H}\right)_{3} . \mathrm{H}_{2} \mathrm{O}$

4. $\left.\mathrm{Fe}_{6}\left(\mathrm{H}_{2} \mathrm{PO}_{2}\right)_{14} \mathrm{SO}_{4}\left(\mathrm{SO}_{4} \mathrm{H}\right)_{2}, 24 \mathrm{H}_{2} \mathrm{O}\right\}$ pulverförmigin), weiß.

5. $\mathrm{Fe}_{6}\left(\mathrm{H}_{2} \mathrm{PO}_{2}\right)_{13} \mathrm{SO}_{4 / 2}\left(\mathrm{SO}_{4} \mathrm{H}\right)_{4} \cdot 30 \mathrm{H}_{2} \mathrm{O}$

6. $\mathrm{Fe}_{3}\left(\mathrm{H}_{2} \mathrm{PO}_{2}\right\rangle_{7}\left(\mathrm{ClO}_{4}\right)_{2} \cdot 1 /{ }_{2} \mathrm{H}_{3} \mathrm{PO}_{2} \cdot 18 \mathrm{H}_{2} \mathrm{O}$ mikrokristallinisch $\left.{ }^{1}\right)$, sehr blaBrot.

7. $\left.\mathrm{Fe}_{8}\left(\mathrm{H}_{2} \mathrm{PO}_{2}\right)_{15}\left(\mathrm{SbCl}_{8}\right)_{3}{ }^{2}\right) \cdot 18 \mathrm{H}_{2} \mathrm{O}$ sehr blaßgelb, mikrokristallinisch.

8. $\mathrm{Fe}_{5}\left(\mathrm{H}_{2} \mathrm{PO}_{2}\right)_{9} \mathrm{Cl}_{6} \cdot 12 \mathrm{H}_{2} \mathrm{O}$ kleine, gelbe Oktaeder.

9. $\mathrm{Fe}_{4}\left(\mathrm{H}_{2} \mathrm{PO}_{2}\right)_{8} \mathrm{Cl}_{4} \cdot 2 \mathrm{H}_{3} \mathrm{PO}_{2} \cdot 10 \mathrm{H}_{2} \mathrm{O}$ plattige, blaßgelbe Kristalle.

10. $\mathrm{Fe}_{4}\left(\mathrm{H}_{2} \mathrm{PO}_{2}\right)_{8} \mathrm{Br}_{4} \cdot 12 \mathrm{H}_{2} \mathrm{O}$ kristallinisch, dunkelrotbraun.

Derartige Verbindungen erhielten wir fernerhin bei bestimmten Mengen- und Konzentrationsverbältnissen aus Natriumbypophosphit und Eisensalz (siehe experimenteller Teil, S. 34 ff.):

11. $\mathrm{Fe}_{6}\left(\mathrm{H}_{2} \mathrm{PO}_{2}\right)_{13}\left(\mathrm{SO}_{4}\right)_{2}(\mathrm{OH}) \cdot 24 \mathrm{H}_{2} \mathrm{O}$

12. $\left.\mathrm{Fe}_{6}\left(\mathrm{H}_{2} \mathrm{PO}_{2}\right)_{13} \mathrm{Cl}_{2}(\mathrm{OH})_{3} \cdot 15 \mathrm{H}_{2} \mathrm{O}\right\}$ weißliche Pulver.

13. $\mathrm{Fe}_{8}\left(\mathrm{H}_{2} \mathrm{PO}_{2}\right)_{15}\left(\mathrm{NO}_{3}\right)_{2}(\mathrm{OH}) \cdot 24 \mathrm{H}_{2} \mathrm{O}$

14. $\mathrm{Fe}_{5}\left(\mathrm{H}_{2} \mathrm{PO}_{2}\right)_{8} \mathrm{Cl}_{7}, 6 \mathrm{H}_{2} \mathrm{O}$ dunkelgelbes Pulver.

1) Siehe hierüber weiter unten S. 23.

2) Rest der Hexachloroantimonsäure, [SbCl $]_{0} \mathrm{H}_{4} 4^{1}{ }_{2} \mathrm{H}_{2} \mathrm{O}$, R. Whindard und Hans Schum, Z. anorg. Chem. 74 (1905), 37. 
An den Formeln sieht man fürs erste, daß die meisten der Verbindungen 3 Eisenatome enthalten oder ein Multiplum von 3. Nur vier enthalten 4 oder 5 Eisenatome, aber diese sind durchweg gelb oder, wie das Bromid, braun gefärbt, woraus hervorgeht, daß das Halogen an Eisen gebunden sein mu $B$. Hierbei ist die naheliegendste Annahme die, daB es sich um Eisenchloro- bzw. Eisenbromoanionen handelt. Zieht man die zu diesen gehörigen Eisenatome $a b$, so bleiben auch in diesen Fällen 3 Eisenatome übrig (siehe näheres weiter unten, S. 18).

Schon lieser Umstand, und die weitere Tatsache, daß alle anderen von uns erhaltenen, weiter unten zu beschreibenden Ferrihypophosphite mit oder ohne anorganischen Säurerest 3 Eisenatome oder ein Multiplum, davon im Molekül enthalten, legt die Vermutung nahe, daß allen diesen Verbindungen, wie den in der Einleitung angeführten Ferrisalzen organischer Säuren, ein komplexes Kation zugrunde liegt, welches wie jene 3 Eisenatome enthält. Für einen solchen Komplex spricht besonders auch die große Beständigkeit der Verbindungen (siehe hierüber weiter unten, S. 23), die schon am gewöhnlichen Ferrihypophosphit (S. 15) auffiel, wie ihre Existenz überhaupt. Die Zahl der zum Komplexgehörenden Hypophosphitreste ergibt sich aus der Zusammensetzung derjenigen Verbindungen, die die geringste Zahl dieser Reste enthalten. Es sind dies die Sulfate Nr. 3,5 und 11 und das Chlorid Nr. 12. Diese enthalten 6 Hypophosphitreste auf 3 Eisenatome bzw. 13 solche auf 6 Eisenatome. Alle anderen Salze enthalten mehr dieser Reste, keines weniger. Hieraus ergibt sich, daß das komple xe $K a$ tion wie dasjenige der organischen Säuren 6 Hy pophosphitres te enthält oder, mit anderen Worten, daß den Salzen die dreisäurige Triferri-hexahypophos phitobase zugrunde liegt:

$$
\left[\mathrm{Fe}_{3}\left(\mathrm{H}_{2} \mathrm{PO}_{3}\right)_{6}\right](\mathrm{OH})_{3} \text {. }
$$

Hiernach ergeben sich für die angeführten Verbindungen die folgenden Konstitutionsformeln :

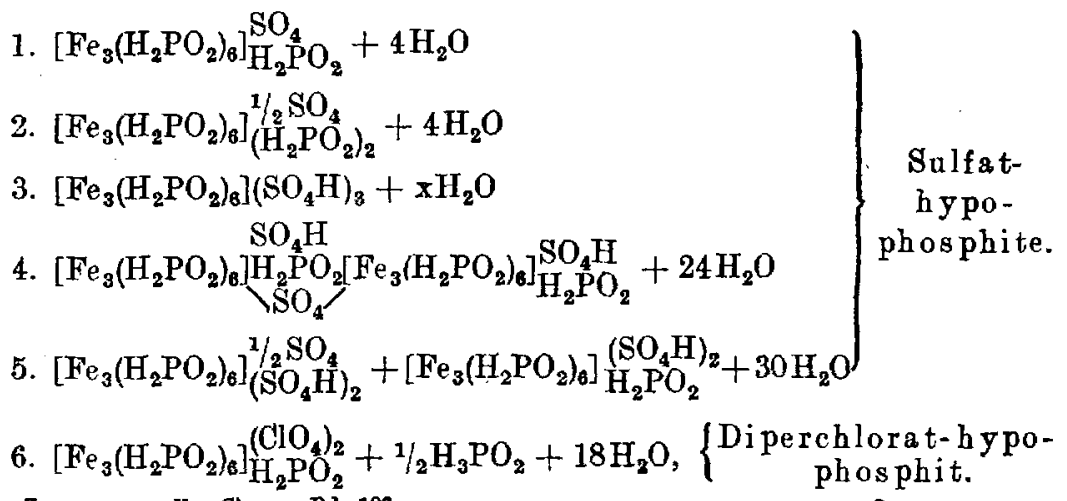

z. anorg. v. allg. Chem. Bd. 106 . 
$\left.\begin{array}{l}\left.\text { 7. }\left[\mathrm{Fe}_{3}\left(\mathrm{H}_{2} \mathrm{PO}_{2}\right)_{8}\right]\right]_{\mathrm{H}_{2} \mathrm{PO}_{2}}^{\left(\mathrm{SbCl}_{6}\right)_{2}} \\ \left.+\left[\mathrm{Fe}_{3}\left(\mathrm{H}_{2} \mathrm{PO}_{2}\right)_{6}\right]\right]_{\left(\mathrm{H}_{2} \mathrm{PO}_{2}\right)_{2}}^{\left(\mathrm{SbCl}_{6}\right)}+18 \mathrm{H}_{2} \mathrm{O}\end{array}\right\} \begin{gathered}\text { Hexachloroantimoniat- } \\ \text { hy pophosphit. }\end{gathered}$

Die gelb gefärbten, chlorhaltigen Salze Nr. 8, 9 und 14 und das tiefbraune bromhaltige Nr. 10 enthalten, wie aus ihrer Farbe zu schlieBen ist, Eisenchloro-bzw. Eisenbromoanionen, und zwar Nr. 9 und 10 ein Tetrachloro-(bromo-)ferrianion:

9. $\left[\mathrm{Fe}_{3}\left(\mathrm{H}_{2} \mathrm{PO}_{2}\right)_{8}\right]_{\left(\mathrm{H}_{2} \mathrm{PO}_{2}\right)_{2}}^{\left[\mathrm{FeCl}_{4}\right]_{3}}+2 \mathrm{H}_{3} \mathrm{PO}_{2}+10 \mathrm{H}_{2} \mathrm{O}\left\{\begin{array}{c}\text { Tetrachloroferriat- } \\ \text { dihypophosphit; }\end{array}\right.$

10. $\left[\mathrm{Fe}_{3}\left(\mathrm{H}_{2} \mathrm{PO}_{2}\right)_{6}\right]\left(\mathrm{H}_{2} \mathrm{PO}_{2}\right)_{2}+12 \mathrm{H}_{2} \mathrm{O}$, Tetrabromoferriat-dihypophosphit.

Bei Nr. 8 und Nr. 14 muB man gemischte Chloro-hypophosphitoferriAnionen annehmen, wobei es für die Verteilung der Säurereste auf die einzelnen Anionen mehrere Möglichkeiten gibt. Wir bevorzugen die folgende, weil auch bei anderen Salzen jeweils ein Hypophosphitrest im Anion sich befindet:

8. $\left[\mathrm{Fe}_{3}\left(\mathrm{H}_{2} \mathrm{PO}_{2}\right)_{6}\right]\left[\begin{array}{c}{\left[\mathrm{Fe}_{\mathrm{H}_{2}}^{\mathrm{Cl}_{3}} \mathrm{PO}_{2}\right.} \\ \mathrm{H}_{2} \mathrm{PO}_{2}\end{array}\right]_{2}+12 \mathrm{H}_{2} \mathrm{O}\left\{\begin{array}{c}\text { Trichlorohypophosphitoferriat- } \\ \text { hypophosphit. }\end{array}\right.$

14. $\left[\mathrm{Fe}_{3}\left(\mathrm{H}_{2} \mathrm{PO}_{2}\right)_{6}\right]\left[\begin{array}{c}{\left[\mathrm{FeCl}_{4}\right]} \\ \mathrm{Fe}_{3}^{\mathrm{Cl}_{3}} \\ \mathrm{H}_{2} \mathrm{PO}_{2} \\ \mathrm{H}_{2} \mathrm{PO}_{2}\end{array}\right]+6 \mathrm{H}_{2} \mathrm{O} \quad\left\{\begin{array}{c}\text { Tetrachloroferriat-trichloro- } \\ \text { hypophosphitoferriat- } \\ \text { hypophosphit. }\end{array}\right.$

Derartige Chloroacido-Anionen bildet das Eisen auch sonst. Z. B. wurden früher Chloroacetato- und Chlorohydroxo-Anionen beobachtet:

$$
\left.\left.\left.\left[\mathrm{Fe}_{\left(\mathrm{CH}_{3} \mathrm{COO}\right)_{2}}^{\mathrm{III}}\right]^{\mathrm{Cl}_{2}}\right) ;\left[\mathrm{Fe}_{\left(\mathrm{CH}_{3} \mathrm{COO}\right)_{3}}^{\mathrm{Cl}}\right]{ }^{1}\right) ;\left[\mathrm{Fe}_{(\mathrm{OH})_{2}}^{\mathrm{Cl}_{2}}\right] \cdot{ }^{2}\right)
$$

Das Salz Nr. 8 ist ausgezeichnet durch seine Kristallisationskraft, seine Kristallform (Oktaeder) und sein großes Bildungsbestreben. Es gehört zu den charakteristischsten und schönsten der ganzen Reihe. Da man es aus allen anderen Hypophosphiten durch Einwirkung von konzentrierter Salzsäure erhält (s. experimenteller Teil, S. 31), kann es zum Nachweis der Hexahypophosphitotriferri-Base überhaupt dienen.

Die noch übrigen drei Salze Nr. 11, 12 und 13 sind basisch, sie enthalten daher Hydroxylgruppen. Diese können, da sich die Verbindungen aus schwach saurer Lösung ausscheiden, nicht Hydroxylionen im Anion sein. Vielmehr gehören sie zum Komplex, und zwar sind es Hydroxog1 uppen im Wernerschen Sinne, da sie mit Säuren (unter Bildung von

1) R. Wernland und Chr. Beck, Z. anorg. Chem. 80 (1913), 421.

2) R. Whinlakd und A. HrRz, Ann. Chem. 400 (1913), 234. 
Aquoverbindungen) zu reagieren vermögen, im Gegensatz zu den gleichfalls zum Komplex gehörenden ol-Gruppen, welche hierzu nicht imstande sind. Die Verbindungen sind hiernach:

$$
\begin{aligned}
& \text { 11. }\left[\mathrm{Fe}_{3}\left(\mathrm{H}_{2} \mathrm{PO}_{2}\right)_{8}\right] \underset{\mathrm{H}_{2} \mathrm{PO}_{2}}{\left(\mathrm{SO}_{2}\right)_{2}}\left[\mathrm{Fe}_{3}\left(\mathrm{H}_{2} \mathrm{PO}_{2}\right)_{8}\right]+24 \mathrm{H}_{2} \mathrm{O} \text {; } \\
& \text { 12. } \left.\left.\left[\mathrm{Fe}_{3} \underset{(\mathrm{OH})}{\left(\mathrm{H}_{2} \mathrm{PO}_{2}\right)_{8}}\right] \underset{\mathrm{H}_{2} \mathrm{PO}_{2}}{\mathrm{Cl}_{2}}\left[\mathrm{Fe}_{3}(\mathrm{OH})_{2}\right)_{2} \mathrm{H}_{2} \mathrm{PO}_{2}\right)_{6}\right]+15 \mathrm{H}_{2} \mathrm{O} \text {; } \\
& \text { 13. }\left[\mathrm{Fe}_{3}\left(\mathrm{H}_{2} \mathrm{PO}_{2}\right)_{6}\right] \underset{\left(\mathrm{NO}_{3}\right)_{2}}{\left(\mathrm{H}_{2} \mathrm{PO}_{3}\right)_{3}}\left[\mathrm{Fe}_{3}\left(\mathrm{H}_{2} \mathrm{PO}_{2}\right)_{8}\right]+24 \mathrm{H}_{2} \mathrm{O} \text {. }
\end{aligned}
$$

Wie ersichtlich, bildet dieses Hexahypophosphitotriferri-Kation mit Vorlie be neutrale und saure Salze, im Gegensatz zu den analogen Kationen der organischen Monocarbonsäuren, welche in erster Linie primäre Salze geben. In den meisten Fällen s'heint ferner noch ein Hypophosphitrest im Anion festgehalten zu werden.

Im folgenden teilen wir zuerst noch eine andere Darstellungsmethode für derartige Salze der Triferrihexahypophosphito-Base mit. Hierauf werden wir uns den Ferrihypophosphiten selbst zuwenden, von denen wir außer dem von Rose dargestellten (S. 15) noch mehrere andere erhalten konnten. Endlich beschreiben wir die Eigenschaften aller dieser Verbindungen mit dem Hexahypophosphitotriferri-Kation.

Ebenso wie man durch Einwirkung von anorganischen Säuren auf Ferrihypophosphite Salze der Hypophosphitobase bekommt, erhält man diese auch bei der Einwirkung von unterphosphoriger Säure auf Ferrisalze. Indessen gelangt man auf diesem Wege nicht zu ganz einheitlichen Stoffen, selbst beim Arbeiten mit fast genau denselben Mengen Ferrisalz und unterphosphoriger Säure erhält man Salze zwar ähnlicher, aber nicht gleicher Zusammensetzung. Die analytischen Werte jedoch liegen so, daß das Verhältnis Eisen : unterphosphoriger Säure : anorganischem Säurerest ganzzahlig wird, wenn Eisen gleich 3 oder einem Multiplum von 3 gesetzt wird. Darüber, daß diese Verbindungen auch das Triferrihe xahypophosphito-Kation enthalten, kann biernach sowie nach ihren Eigenschaften kein Zweifel sein. Die Möglichkeit, solche Verbindungen aus Ferrisalzen starker anorganischer Säuren durch die verhältnismäßig schwache unterphosphorige Säure überhaupt abzuscheiden, beweist, da $B$ die Neigung des dreiwertigen Eisens, mit unterphosphoriger Säure dieses komplexe Kation zu bilden, eine sehr große ist.

Man erbält z. B. aus Ferriperchlorat und unterphosphoriger Säure bei bestimmten Konzentrationsverhältnissen (s. experimenteller Teil, S. 36 ff.) Körper, die man als Gemenge folgender Perchlorate der Hexahypophosphitotriferri-Base ansehen kann: 
15.

$$
\begin{aligned}
{\left[\mathrm{Fe}_{3}\left(\mathrm{H}_{2} \mathrm{PO}_{2}\right)_{6}\right] \underset{\mathrm{H}_{2} \mathrm{PO}_{2}}{\left(\mathrm{ClO}_{2}\right)_{2}}+\underset{\mathrm{xH}_{2} \mathrm{O} \text { und }}{ }\left[\mathrm{Fe}_{3}\left(\mathrm{H}_{2} \mathrm{PO}_{2}\right)_{6}\right]_{2} \underset{\left(\mathrm{H}_{2} \mathrm{PO}_{2}\right)_{3}}{\left(\mathrm{ClO}_{4}\right)_{3}} } \\
+\underset{\text { (erste Reihe); }}{2 \mathrm{H}_{2} \mathrm{PO}_{2}+\mathrm{HH}_{2} \mathrm{O}}
\end{aligned}
$$

$$
\left.\left[\mathrm{Fe}_{3}\left(\mathrm{H}_{2} \mathrm{PO}_{2}\right)_{6}\right]_{\left(\mathrm{H}_{2} \mathrm{PO}_{2}\right)_{2}}+\mathrm{xH}_{2} \mathrm{O} \text { und }\left[\begin{array}{l}
\mathrm{Fe}_{3}\left(\mathrm{H}_{2} \mathrm{PO}_{2}\right)_{6} \\
(\mathrm{OH})_{2}
\end{array}\right] \mathrm{H}_{2} \mathrm{PO}_{2}+x \mathrm{H}_{2} \mathrm{O}^{2}\right)
$$

Aus Ferrisulfat und unterphosphoriger Säure erhält man in ähnlicher Weise Sulfathypophosphite der Base, die ein Gemenge folgender Salze vorstellen:

$\left[\mathrm{Fe}_{3} \underset{(\mathrm{OH})}{\left(\mathrm{H}_{2} \mathrm{PO}\right)_{6}}\right] \mathrm{SO}_{4}+x \mathrm{H}_{2} \mathrm{O}$ und $\left[\mathrm{Fe}_{3}\left(\mathrm{H}_{2} \mathrm{PO}_{2}\right)_{6}\right]_{2}\left(\mathrm{H}_{2} \mathrm{PO}_{2}\right)_{4}+\mathrm{H}_{3} \mathrm{PO}_{2}+x \mathrm{H}_{2} \mathrm{O}$.

Ferrichlorid und unterphosphorige Säure ergeben ebenso Salze, die in ihrer Zusammensetzung zwischen den Extremen liegen:

$$
\left.\left[\mathrm{Fe}_{3}\left(\mathrm{H}_{2} \mathrm{PO}_{2}\right)_{6}\right]_{\mathrm{H}_{2} \mathrm{PO}_{2}}^{\mathrm{Cl}_{2}}+\mathrm{xH}_{2} \mathrm{O} \text { und }\left[\mathrm{Fe}_{3} \underset{(\mathrm{OH})}{\left(\mathrm{H}_{2} \mathrm{PO}_{2}\right)_{6}}\right]\left(\mathrm{H}_{2} \mathrm{PO}_{2}\right)_{2}+x_{2} \mathrm{O} .{ }^{2}\right)
$$

Noch andere Verbindungsreihen dieser Art könnte man, auch mit anderen Ferrisalzen, je unter bestimmten Versuchsbedingungen, erhalten. Alle auf dies Weise dargestellten Salze der Base gleichen den oben beschriebenen ganz im Ausseben und Verhalten.

Was sodann die Ferrihypophosphite selbst betrifft, so gibt, wie schon oben S. 15 erwähnt, H. Rose an, daß man durch Einwirkung von unterphospboriger Säure auf Ferrihydroxyd ein weißes Salz bekommt. Diese Darstellung von Ferrihypophosphit erschien jedoch, wie oben S. 15 schon ausgeführt, ungeeignet, und wir haben daher versucint, Ferrihypophosphit auf andere Weise darzustellen. Wir fanden, daß dies sehr leicht durch Umsetzung von Ferrichlorid mit Natriumhypophosphit in wäBriger Lösung möglich ist. Hierbei erhält man, je nach den Arbeitsbedingungen - verschiedene Konzentration, heiß oder kalt, Zusatz der Ferrichloridlösung zum Natriumhypophosphit oder umgekehrt (siehe experimenteller Teil) - drei verschiedene Ferrihypophosphite:

18. $\left.\mathrm{Fe}_{3}\left(\mathrm{H}_{2} \mathrm{PO}_{2}\right)_{8}(\mathrm{OH})+\mathrm{xH}_{2} \mathrm{O}^{3}\right)$;

mit dem Verhältnis $\mathrm{Fe}: \mathrm{H}_{2} \mathrm{PO}_{2}=3: 8$;

19. $\mathrm{Fe}_{6}\left(\mathrm{H}_{2} \mathrm{PO}_{2}\right)_{15}(\mathrm{OH})_{3}+\mathrm{xH}_{2} \mathrm{O} ; \mathrm{Fe}: \mathrm{H}_{2} \mathrm{PO}_{2}=6: 15$;

20. $\mathrm{Fe}\left(\mathrm{H}_{2} \mathrm{PO}_{2}\right)_{3} ; \mathrm{Fe}: \mathrm{H}_{2} \mathrm{PO}_{2}=1: 3(=3: 9)$.

Von diesen ist das erste mit dem Verhältnis Eisen: unterphosphoriger Säure $=3: 8$ dasjenige, das man für gewöhnlich erhält, daher diente es uns, wie schon angegeben, meistens als Ausgangsmaterial für

1) Basisches Ferrihypophosphit, $\mathrm{Fe}: \mathrm{H}_{2} \mathrm{PO}_{2}=3: 7$, s. näheres unten $\mathrm{S} .22 \mathrm{ff}$.

2) Basisohes Ferrihypophosphit, $\mathrm{Fe}: \mathrm{H}_{2} \mathrm{PO}_{2}=3: 8$, s. unten.

3) Uther den Wassergehalt dieser Salıe siehe unten im experimentellen Teil S. 40 ff. und S. 25. 
die weitere Untersuchung. Aus ihm, sowie aus allen bisher angeführten Verbindungen, auch denen mit anderen Säuren im Anion, erhält man durch Behandlung mit heißem Wasser infolge von Hydrolyse das neue Ferrihypopliosphit

$$
\text { 21. } \mathrm{Fe}_{3}\left(\mathrm{H}_{2} \mathrm{PO}_{2}\right)_{7}(\mathrm{OH})_{2}+\mathrm{xH}_{2} \mathrm{O} \text {; mit dem Verhältnis } \mathrm{Fe}: \mathrm{H}_{2} \mathrm{PO}_{2}=3: 7 \text {. }
$$

Hierbei wird ein Teil des dreiwertigen Eisens zu zweiwertigem reduziert und Phosphorsäure gebildet. Das erhaltene Ferrihypophosphit ist aber frei von Phosphorsäure (die Molybdänsäurereaktion ist negativ), auch enthält es kein zweiwertiges Eisen. Phosphorsäure und zweiwertiges Eisen befinden sich vielmehr ausschlieblich in der überstehenden sauren Flüssigkeit.

Dagegen entsteht durch längere Einwirkung von kaltem Wasser auf alle genannten Salze ein Ferrihypophosphit mit dem Verhältnis $\mathrm{Fe}: \mathrm{H}_{2} \mathrm{PO}_{2}$ $=1: 2$ :

$$
\text { 22. } \mathrm{Fe}\left(\mathrm{H}_{2} \mathrm{PO}_{2}\right)_{2}(\mathrm{OH}) \text {; }
$$

es ist bemerkenswerterweise noch basischer als das vorhergehende. Bei der Darstellung löst sich ein Teil des Ferrihypophosphits in dem Wasser und aus dieser Lösung scheidet sich beim Verdunsten das Salz 3 : 7 besonders schön kristallinisch aus.

Dasselbe basische Hypophosphit bekommt man nun eigentümlicherweise auch beim Behandeln des Ferrihypophosphits mit unterphosphoriger Säure. Eigentlich hätte man erwarten müssen, daß die basischen Hypophosphite hierbei in weniger basische oder in neutrales Hypophosphit

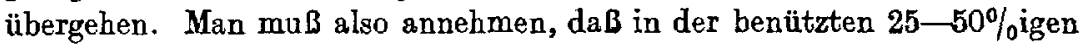
unterphosphorigen Säure immer noch Hydrolyse stattfindet. In der Tat fanden wir auch, daB bei ıangere Digestion mit unterphosphoriger Säure, wobei diese konzentrierter wird, weniger basisches oder neutrales Ferrihypophosphit sich bildet.

Auch Löslichkeitsverschiedenheiten können diese merkwürdigen Tatsachen erklären: das Salz 1 : 2 scheint nämlich schwerer löslich als irgendein anderes weniger basisches Ferribypophosphit zu sein und so eine gewisse Sonderstellung einzunehmen (vgl. auch unten S. 22). Die in der Flüssigkeit noch gelösten, weniger basischen Salze erscheinen daher erst später durch Verdunsten derselben, wie auch das Salz 3: 7 bei der vorher erwähnten Darstellung.

Diese Ferrihypophosphite bilden weißliche, sehr feine Pulver, mit einem mehr oder weniger deutlichen Stich ins Rötliche. Sie gleichen hierin und auch sonst vollständig whlreichen oben angeführten Verbindungen des dreiwertigen Eisens mit unterphosphoriger Säure und anderen Säuren ${ }^{1}$ ), in denen sich das Hexahypophosphito-Kation befindet. Aus

1) In der Farbe natürlich nicht denjenigen mit Eiscuchloro-(bromo)zäure im Anion. 
diesem Grunde, und besonders auch deshalb, weil mehrere Ferrihypophosphite wieder die Dreizahl für die Eisenatome aufweisen, ist auch bei diesen Salzen die Hexahy pophosphitotriferri-Base anzunehmen. Das Salz 3:7 ist hiernach das Monohypophosphit der Base:

$$
\text { 21. }\left[\mathrm{Fe}_{3}(\mathrm{OH})_{2} \mathrm{H}_{2} \mathrm{PO}_{8}\right) \mathrm{H}_{2} \mathrm{PO}_{2}
$$

das Salz $3: 8$ das Dihypophosphit derselben

$$
\text { 18. }\left[\mathrm{Fe}_{3}(\mathrm{OH})=\left(\mathrm{H}_{2} \mathrm{PO}_{2}\right)_{6}\right]\left(\mathrm{H}_{2} \mathrm{PO}_{2}\right)_{2} \text {; }
$$

das Salz 6 : 15 stellt eine Doppelverbindung der beiden vorhergehenden dar:

$$
19\left[\mathrm{Fe}_{3} \underset{(\mathrm{OH})}{\left(\mathrm{H}_{2} \mathrm{PO}_{2}\right)_{6}}\right]\left(\mathrm{H}_{2} \mathrm{PO}_{2}\right)_{2}+\left[\mathrm{Fe}_{3}(\mathrm{OH})_{2}\left(_{2} \mathrm{PO}_{2}\right)_{6}\right] \mathrm{H}_{2} \mathrm{PO}_{2} \text {, }
$$

wie solche auf diesem Gebiete nicht selten beobachtet wurden. Wir erwähnen die analoge Hexabenzoatotriferri-Kationverbindung:

$$
\left.\left[\mathrm{Fe}_{3} \underset{(\mathrm{OH})}{\left(\mathrm{C}_{6} \mathrm{H}_{5} \mathrm{COO}\right)_{6}}\right]\left(\mathrm{C}_{6} \mathrm{H}_{5} \mathrm{COO}\right)_{2}+\left[\mathrm{Fe}_{3} \underset{(\mathrm{OH})_{2}}{\left(\mathrm{C}_{6} \mathrm{H}_{5} \mathrm{COO}\right)_{6}}\right] \mathrm{C}_{6} \mathrm{H}_{5} \mathrm{COO} .{ }^{\mathrm{l}}\right)
$$

Das oben $\mathbf{S} .15$ angeführte Hypophosphit $1: 3$ kann hiernach nicht ein einfaches normales Ferrihypophosphit sein, sondern auch dieses muß die Base mit den drei Eisenatomen enthalten. Es ist somit das Trih y pophosphit derselben:

$$
\text { 20. }\left[\mathrm{Fe}_{3}\left(\mathrm{H}_{2} \mathrm{PO}_{2}\right)_{6}\right]\left(\mathrm{H}_{2} \mathrm{PO}_{2}\right)_{3} \text {. }
$$

Es bleibt noch die Konstitution des basischen Salzes 1:2 zu erörtern. Schon seine Bildungsweise aus den Ferrihypophosphiten, die das komplexe Kation enthalten, macht es wahrscheinlich, daß auch dieses Salz von einem solchen sich ableitet, dazu kommen die durchaus gleichen Eigenschaften. Man ist also berechtigt, auch in ihm einen solchen Komplex, der 3 Eisenatome enthält, anzunehmen. Die Bruttoformel der Verbindung ist hiernsch

$$
\mathrm{Fe}_{3}\left(\mathrm{H}_{2} \mathrm{PO}_{2}\right)_{6}(\mathrm{OH})_{3} \text {. }
$$

Man könnte sie als die freie Hexahypophosphitotriferri-Base selbst ansehen. Dagegen spricht jedoch, daß man diese Verbindung aus saurer Lösung bekommt. Sodann sind bei den Monocarbonsäuren wiederholt Verbindungen der analogen Zusammensetzung erhalten worden, z. B. ein Ferribenzoat

$$
\left.\mathrm{Fe}_{3}\left(\mathrm{C}_{6} \mathrm{H}_{5} \mathrm{COO}\right)_{6}(\mathrm{OH})_{3} .1 \mathrm{H}_{2} \mathrm{O} .{ }^{1}\right)
$$

Bei diesen ist es aber überaus wahlscheinlich, daß sie ein Kation enthalten, welches aus 3 Atomen Eisen und 5 Säureresten besteht. Dies wird auch für das obige Hypophosphit zutreffen, und wir sehen es

1) R. WEnnland und A. HERz, Ber. deutsch. chem. Ges. 45 (1912), 2667. 
als ein Monohypophosphit einer PentahypophosphitotriferriBase an:

$$
\text { 22. }\left[\mathrm{Fe}_{3}(\mathrm{OH})_{3}{\left(\mathrm{H}_{2} \mathrm{PO}_{2}\right)_{5}}_{3}\right] \mathrm{H}_{2} \mathrm{PO}_{2} \text {. }
$$

Für diese neue Base spricht auch die oben angedeutete Sonderstellung dieses Salzes gegenüber den anderen Ferrihypophosphiten, die seine eigenartige Bildungsweise erklärt (S.21).

Wir fügen hinzu, daß bei organischen Säuren komplexe Kationen mit noch weniger Säureresten beobachtet wurden, z. B.:

$$
\left.\left[\begin{array}{c}
\mathrm{Fe}_{3} \stackrel{\left(\mathrm{C}_{6} \mathrm{H}_{5} \mathrm{COO}\right)_{3}}{\mathrm{O}} \\
(\mathrm{OH})_{3}
\end{array}\right] \mathrm{C}_{6} \mathrm{H}_{5} \mathrm{COO} .{ }^{1}\right)
$$

Aus alledem ergibt sich, daß alle Ferrihypophosphite, wie die Ferrisalze organischer Säuren, nicht einfache Salze im Sinne der bisherigen Formulierung vorstellen, sondern wesentlich komplizierter konstituiert sind, insofern sie komplexe mehrkernige Kationen enthalten. Uber den Bau derartiger Kationen hat W FRNER (in seinen Neueren Anschauungen, 3. Aufl., S. 282) eine Vorstellung entwickelt, indessen gibt es hier mehrere Möglichkeiten, zwischen denen man experimentell heute noch nicht entscheiden kann.

Wir gehen nunmehr zur Beschreibung der Eigenschaften dieser Verbindungen über.

Was zuerst an einer Anzahl der Verbindungen auffällt, ist, daß die sauren wie das Perchlorat (S. 17, Nr.6) und die Sulfate (S. 17, Nr. 3-5) aus der Mutterlauge herausgenommen und auf Ton getrocknet oft sehr rasch von dem ursprünglich pulvrigen, mehr oder weniger kristallinisehen Zustand in eine zusammenhängende, zähe, sogar etwas klebrige Masse übergehen. Dieser Vorgang findet an der Luft sowohl, wie über Chlorcalcium oder Schwefelsäure statt. Hierbei tritt die zuerst nur blaßrötliche Färbung der Verbindungen deutlicher hervor, da die Masse allmählich glasig durchscheinend wird. Bei längerem Liegen über Chlorcalcium erhärtet sie und zeigt einen muscheligen Bruch. Manchmal findet diese Veränderung schon bei längerem Liegen der Körper unter der Mutterlauge statt.

Da besonders die sauren und übersauren Salze diese Umwandlung erleiden, möchten wir sie auf $\mathrm{Hydrolyse}$ zurückführen. Infolgedessen wird unterphosphorige Säure abgespalten, die selbst eine zähe klebrige Substanz ist und so diese Veränderung hervorrufen kann. Das die Hydrolyse bewirkende Wasser entstammt den Körpern selbst, welche sehr wasserreich sind (s. unten).

Derartige saure und übersaure Salze soleher komplexer Kationen er-

1) R. W Ennland und A. Herz, Ber. deutzeh, chem. Ges. 45 (1912), 2670; s. auch R. WETNLAND und E. BÜTTNER, Z. anorg. Chem. 75 (1912), 316. 
leiden auch sonst leicht Hydrolyse, wie z. B. die sauren Acetate der Pentaund Triacetatotrichromi-Base.

$$
\begin{aligned}
& {\left[\mathrm{Cr}_{3}{ }_{(\mathrm{CH}}^{(\mathrm{OH})_{2}}\right)_{5}} \\
& \left(\mathrm{CH}_{3} \mathrm{COO}\right)_{2}+2 \mathrm{CH}_{3} \mathrm{COOH}+3 \mathrm{H}_{2} \mathrm{O} ; \\
& \left.\left[\mathrm{Cr}_{3} \underset{(\mathrm{OH})_{2}}{\left(\mathrm{CH}_{3} \mathrm{COO}\right)_{3}}\right]\left(\mathrm{CH}_{3} \mathrm{COO}\right)_{4}+2 \mathrm{CH}_{3} \mathrm{COOH} .{ }^{1}\right)
\end{aligned}
$$

Alle (stöchiometrisch) neutralen und basischen Salze erleiden diese Unwandlung nicht, die reinen Hypophosphite eingeschlossen. Eine Ausnahme hiervon macht zuweilen das als Ausgangsmaterial benutzte basische Hypophosphit 3:8. Bei diesem kommt der Ubergang in die amorphe Masse vor, wenn es nicht sorgfältig von der Mutterlauge, die unterphosphorige Säure enthält, befrejt wurde.

Beim Kochen in der Fällungsflüssigkeit erleiden übrigens alle Hypophosphite eine merkwürdige Veränderung in ihrer Beschaffenheit, indem sie sich infolge der Hitze zu einer zähen Masse zusammenballen, die sich, wenn sie weich genug wird, in lange Fäden ausziehen läBt. Diese Eigenschaft mag bei manchen Körpern, die gerade in der Hitze erhalten wurden und selbst nicht sauer sind, Ursache ihres besonderen Verhaltens sein (z. B. die Salze Nr. 15, 16, 17, exper. Teil, S. 36).

Die meisten Verbindungen enthalten Wasser, und zwar einige ziemlich viel. So das Perchlorathypophosphit (Nr. 6) 18, das gelbe oktaedrische Chloroferriat' (Nr. 8) 12 Moleküle. Wasserfrei ist dagegen das (in der Hitze oder nach Rose) dargestellte normale Hypophosphit Nr. 20. Die hohen Wassergehalte sind insofern auffallend, als $\operatorname{man}$ bei in Wasser schwer löslichen Stoffen, wie diese es sind, einen solchen Wassergehalt sonst nicht antrifft. Dieses Wasser ist allerdings sehr locker gebunden, manche Salze verwittern schon rasch an der Luft, so daB es oft nicht möglich war, den ursprünglichen Wassergehalt genau festzustellen. Dies fanden wir besonders bei den reinen basischen Hypophosphiten S. 20. Uber Chlorcalcium verlieren sie alle das gesamte Wasser, woraus hervorgeht, daß das Wasser keinen wesentlichen Anteil an der Konstitution der Verbindungen hat. Sehr wasserhaltig sind übrigens auch andere Salze analoger Kationen mit organischen Säuren. So enthält das Chlorid der Hexaacetatotrichromi-Base 8 Moleküle Wasser, das Hexachloroantimoniat 10-Moleküle. ${ }^{2}$ )

An der Luft sind sämtliche Körper unbegrenzt beständig. Wie alle Hypophosphite, so sind auch die in Rede stehenden sämtlich brennbar. Sie entzünden sich beim Erhitzen oder beim Berühren mit einem heißen Magnesiastäbehen.

1) R. Whinlakd und E. BütTher, $Z$. anorg. Chem. 75 (1912), 354, 367.

2) Ber. deutsch. chem. Ges. 42 (1909), 3012, 3016. 
Das Perchlorat ist explosiv. Solange es wasserhaltig ist, explodiert es erst durch Berührung mit einer Flamme. Man kann sogar ein Stückchen der in die zusammenhängende Masse verwandelten Verbindung (s. oben S. 23) ohne Gefahr zwischen den Fingern an eine Flamme halten; es finden dann nur an der erhitzten Stelle kleine Explosionen statt, die sich aber nicht durch das ganze Stückchen fortpflanzen. Ist das Salz aber über Chlorcalcium scharf getrocknet, so kann es schon bei Berührung mit dem Finger unter lautem Knall explodieren. Der Explosionsdru $\mathrm{k}$ ist jedoch nicht groß, denn ein Uhrglas, auf dem die Explosion stattfindet, wird nicht immer zertrümmert. Wir erklären die schlechte Fortpflanzung der Explosion bei dem wasserhaltigen Salze damit, daß die Wassermoleküle eine Pufferwirkung ausüben.

Im Wasser sind die Verbindungen, wie schon erwähnt, sämtlich Bchwer löslich. Nur die ganz frisch dargestellten Ferrihypophosphite sind merklich löslich (s. exper. Teil, S. 41); getrocknet sind sie es nicht mehr. Viele der Salze erleiden durch Wasser weitgehende Hydrolyse, so $\mathrm{daB}$ sie in basische Hypophosphite übergehen (S. 21). Weiter geht aber die Hydrolyse nicht, man bekommt in keinem Fall, wie bei anderen Ferrisalzen, Ferıihydroxyd; so beständig sind diese komplexen Kationen. Die Beständigkeit der Base zeigt sich auch darin, daß die reinen Hypophosphite durch Mineralsäyren, die ziemlich konzentriert sein können (Úberchlorsäure $20 \% \mathrm{ig}$, Salzzäure $38 \%$ ig, Schwefelsäure sogar über $50 \%$ ig usw.), in Salze der Hypophosphito-Base mit jenen Säuren verwandelt werden. Hieraus und aus dem Umstande, daB das dreiwertige Eisen in den Lösungen der Salze in den Mineralsäuren durch die unterphosphorige Säure ers ${ }^{\dagger}$ bei längerem Erhitzen reduziert wird, muß man schließen, da in diesen Lösungen die komplexen Kationen noch vorhanden sind. Hierin ist dieser Komplex der unterphosphorigen Säure denjenigen der organischen Säuren weit überlegen. Diese würden unter diesen Umständen völlig zersetzt. Die Beständigkeit des Komplexes ergibt sich auch aus seiner Bildung bei der Finwirkung der unterphosphorigèn Säure auf Ferrisalze starker anorganischer Säuren (s. oben S. 19). So beständig der Komplex als Base gegen Säuren ist, so empfindlich ist er gegen Basen. Alkalihydroxyde, Alkalikarbonate oder Ammoniak zersetzen ihn sogleich völlig unter Abscheidung von Ferrihydroxyd. Ebenso wird er von Schwefelammon zersetzt.

Damit ist dieses interessante komplexe Kation bei einer anorganischen Säure zum ersten Mal beobachtet worden. Diese enthäl, was vielleicht ron Wichtigkeit ist, wie die Carbonsäuren zwei Sauerstoffatome und ist wie sie einbasisch:

$$
\mathrm{R}-\mathrm{C}<\mathrm{OH}
$$

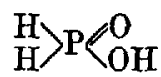




\section{Experimenteller Toil.}

Als Ausgangshypophosphit diente uns ein aus Eisenchlorid und Natriumhypophosphit dargestelltes Präparat, welches, über Chlorcalcium getrocknet, im wesentlichen die Zusammensetzung eines Dihypophosphits der Base zeigt (vgl, oben S. 20):

$$
\left.\left[\mathrm{Fe}_{3}\left(\mathrm{OH}_{2} \mathrm{PO}\right) \mathrm{O}_{2}\right)_{6}\right]\left(\mathrm{H}_{2} \mathrm{PO}_{2}\right)_{2} \text {. }
$$

Zu seiner Darstellung fügt man unter Umrühren eine Lösung von $1 / 10 \mathrm{Mol}$ Ferrichloridhexahydrat $(27 \mathrm{~g})$ in etwa $150 \mathrm{ccm}$ kaltem Wasser zu einer solchen von $3 / 10$ Molen Natriumbypophosphit $\left(32 \mathrm{~g} \mathrm{NaH}_{2} \mathrm{PO}_{2} \cdot \mathrm{H}_{2} \mathrm{O}\right.$ ) in etwa $100 \mathrm{ccm}$ Wasser. Der sogleich entstehende Niederschlag wird durch mäßiges Erhitzen feinpulvrig, dicht und gut filtrierbar. Man wäscht ihn mit wenig kaltem Wasser und trocknet ihn über Chlorcalcium. Weißliches bis blaBrötliches Pulver; wenn feucht oft zusammenklebend (S. 23).

I. $0.2282 \mathrm{~g}$ Substanz: $\left.0.0740 \mathrm{~g} \mathrm{Fe}_{2} \mathrm{O}_{3}{ }^{\mathrm{l}}\right)=22.68 \% \mathrm{Fe}$ und $0.2960 \mathrm{~g}$ $\left.\mathrm{Mg}_{2} \mathrm{P}_{2} \mathrm{O}_{7}{ }^{1}\right)=75.78 \% \mathrm{H}_{2} \mathrm{PO}_{2}$.

II. $0.3470 \mathrm{~g}$ Substanz: $0.0960 \mathrm{~g} \quad \mathrm{Fe}_{2} \mathrm{O}_{3}=19.35 \%$ Fe und $0.3808 \mathrm{~g}$ $\mathrm{Mg}_{2} \mathrm{P}_{2} \mathrm{O}_{7}=64.11 \% \mathrm{H}_{2} \mathrm{PO}_{2}$.

III. $0.3066 \mathrm{~g}$ Substanz: $0.0752 \mathrm{~g} \quad \mathrm{Fe}_{2} \mathrm{O}_{3}=17.15 \%$ Fe und $0.2810 \mathrm{~g}$ $\mathrm{Mg}_{2} \mathrm{P}_{2} \mathrm{O}_{7}=53.55 \% \mathrm{H}_{2} \mathrm{PO}_{3}$.

IV. $0.3110 \mathrm{~g}$ Substanz: $0.1020 \mathrm{~g} \quad \mathrm{Fe}_{2} \mathrm{O}_{3}=22.94 \%$ Fe und $0.3770 \mathrm{~g}$ $\mathrm{Mg}_{2} \mathrm{P}_{2} \mathrm{O}_{7}=70.82 \% \mathrm{H}_{2} \mathrm{PO}_{2}$.

V. $0.3636 \mathrm{~g}$ Substanz: $0.0795 \mathrm{~g} \quad \mathrm{Fe}_{2} \mathrm{O}_{3}=15.29 \% \mathrm{Fe}$ und $0.2862 \mathrm{~g}$ $\mathrm{Mg}_{2} \mathrm{P}_{2} \mathrm{O}_{2}=45.99 \% \mathrm{H}_{2} \mathrm{PO}_{2}$.

Das gibt die Verhältnisse: I. $3 \mathrm{Fe}: 8.58 \mathrm{H}_{2} \mathrm{PO}_{2} ;$ II. $3 \mathrm{Fe}: 8.28 \mathrm{H}_{2} \mathrm{PO}_{2}$; III. $3 \mathrm{Fe}: 8.04 \mathrm{H}_{2} \mathrm{PO}_{2} ;$ IV. $3 \mathrm{Fe}: 7.88 \mathrm{H}_{2} \mathrm{PO}_{2} ;$ V. $3 \mathrm{Fe}: 7.74 \mathrm{H}_{2} \mathrm{PO}_{2}$.

1) Zur Bestimmung des Eisens und der unterphosphorigen Säure in allen diesen Verbindungen wurde die Substanz in etwa $20 \mathrm{ccm}$ etwa $25 \%$ iger Salzsäure gelöst, die Lösung mit ungefähr $3 \mathrm{com}$ Brom versetzt und 3 Stunden auf schwach siedendem Wasserbade erwärmt. In dieser Zeit ist die Oxydation der unterphosphorigen Säure zu Phosphorsäure vollendet. (Diese Oxydation verläuft langsamer als man erwarten sollte, daher ist es nötig, das Brom solange einwirken zu lassen. Dies zeigt sich auch darin, daß konzentrierte Salpetersäure und sogar Königawasser die Oxydation bis zu Phosphorsäure so langsam bewirken, daB sie hierzu ungeeignet sind. Das komplexe Kation wird auch in so starken Säuren nur allmählich zersetzt, wodurch die unterphosphorige Säure der Oxydation zugänglich wird.) Aus der oxydierten Lösung wird das Brom durch Erwärmung vertrieben, und hierauf in ammoniakalischer Lösung mit Schwefelammon Eisen von Phosphorgäure getrennt. 


\section{Sulfate.}

(vgl. oben S. 17.)

1. Hexah ypophos phitotriferri-sulfat-h y pophos phit. $\left.{ }^{1}\right)$

Man übergießt $5 \mathrm{~g}$ des Ausgangshypophosphits mit $10 \mathrm{ccm}$ einer Schwefelsäure, die durch Verdünnung von 1 Vol. konzentrierter Säure mit $11 / 2$ Vol. Wasser erhalten wurde, und läßt in der Kälte stehen. Schon nach einem Tag ist der Bodenkörper in ein sehr feines, hartes, knirschendes Pulver verwandelt. Um aber sicher zu sein, daß die Umwandlung vollständig ist, läßt man etwa 3 Tage stehen, Durch Absaugen und Aufstreichen auf Ton gelingt es, diese feinsandigen Körper vollständig von der Mutterlauge zu befreien. Man kann sie nicht mit Wasser waschen, da sie hierbei Hydrolyse erleiden.

Der luftrockene Körper enthält 4 Moleküle Wasser, welche über Chlorcalcium bald vollständig entweichen. Der im Vergleich zu den anderen Verbindungen geringe Wassergehalt ist wohl auf die Bildung in ziemlich konzentrierter Schwefelsäure zurückzuführen. Weißes Pulver mit einem ganz schwachen Stich ins Rötliche; unter dem Mikroskop bei schwacher Vergrößerung traubige Formen, bei starker Vergrößerung Blättchen von rhombischen oder quadratischem Umriß. Die Verbindung zeigt die oben (S. 23) beschriebene Umwandlung in die amorphe Masse nicht.

$$
\left[\mathrm{Fe}_{3}\left(\mathrm{H}_{2} \mathrm{PO}_{2}\right)_{6}\right]_{\mathrm{H}_{2} \mathrm{PO}_{2}}^{\mathrm{SO}_{4}}+4 \mathrm{H}_{2} \mathrm{O}(791.06) \text {. }
$$

$$
\begin{aligned}
& \text { I. } 0.2470 \mathrm{~g} \text { Substanz: } 0.0753 \mathrm{~g} \mathrm{Fe}_{2} \mathrm{O}_{3}, 0.2434 \mathrm{~g} \mathrm{Mg}_{2} \mathrm{P}_{8} \mathrm{O}_{7} \text {. } \\
& 0.2682 \mathrm{~g} \quad, \quad 0.0811 \mathrm{~g} \mathrm{Fe} \mathrm{O}_{3}, 30.0777 \mathrm{~g} \mathrm{BaSO} \text {. } \\
& \text { II. } 0.5993 \mathrm{~g} \quad, \quad 0.1820 \mathrm{~g} \mathrm{Fe} \mathrm{O}_{3}, 0.5916 \mathrm{~g} \mathrm{Mg}_{2} \mathrm{P}_{2} \mathrm{O}_{7} \text {. } \\
& 0.4820 \mathrm{~g} \quad " \quad 0.1404 \mathrm{~g} \mathrm{Fe}_{2} \mathrm{O}_{3}, 0.1432 \mathrm{~g} \mathrm{BaSO} \text {. } \\
& \text { III. } 0.2160 \mathrm{~g} \quad " \quad 0.0658 \mathrm{~g} \mathrm{Fe}_{2} \mathrm{O}_{3}, 0.2134 \mathrm{~g} \mathrm{Mg} \mathrm{Mg}_{2} \mathrm{O}_{7} \text {. } \\
& 0.2100 \mathrm{~g} \quad " \quad 0.0642 \mathrm{~g} \mathrm{Fe} \mathrm{O}_{3}, 0.0618 \mathrm{~g} \mathrm{BaSO} \text {. } \\
& \text { Berechnet: } \quad \mathrm{Fe} 21.18, \mathrm{H}_{2} \mathrm{PO}_{2} \text { 57.57; } \mathrm{Fe} 21.18, \mathrm{SO}_{4} 12.14 \text {. } \\
& \text { Gefunden: I. ,2 21.33, " 57.57; ,21.16, " 11.92, } \\
& \text { " II. " 21.24, " } \quad 57.67 ; \quad \text { 20.37, ", 12.23, } \\
& \text { " III. ", 21.31, ", 57.71; ", 21.38, ", } 12.11 \text {. } \\
& 0.5360 \mathrm{~g} \text { Substanz verloren über Chlorcalcium } 0.0489 \mathrm{~g} \mathrm{H}_{\mathbf{2}} \mathrm{O} \text {. } \\
& \text { Berechnet: } \mathrm{H}_{2} \mathrm{O} \text { 9.11. } \\
& \text { Gefunden: "9.13. }
\end{aligned}
$$

2. Sulfat-dihypophosphit.

Man löst $3 \mathrm{~g}$ des Ausgangshypophosphits in 5 ecm derselben Schwefelsäure wie bei Nr. 1 durch vorsichtiges, sehr gelindes Erwärmen. ${ }^{3}$ ) Im

1) Die Nummern der Salze in diesem Teil sind dieselben wieim allgemeinen Teil.

2) Zur Bestimmung der Schwefelsäure löst man in etwa $30 \mathrm{ccm} 15 \%$ iger Salzsäure in der Kälte, verdünnt auf etwa $100 \mathrm{ccm}$, fügt kalt überschüssiges Ammoniak hinzu, erwärmt und filtriert. Das Filtrat enthält die gesamte Schwefelsäure. Das Ferrihydroxyd fällt unter diesen Unständen rein und frei von Hypophosphit aus.

3) Damit keine Reduktion des Eisens durch die unterphosphorige Sżure stattfindet. 
Laufe mehrerer Tage scheiden sich kleine blaßrote Kriställchen aus, welche wie der vorhergehende Körper von der Mutterlauge befreit werden. Unter dem Mikroskop aggregierte Blättchen. Sie zeigen die Umwandlungserscheinung nicht (S. 23). Lufttrocken enthält der Körper 4 Moleküle Wasser, welche über Chlorcalcium rasch entweichen.

$$
\left[\mathrm{Fe}_{3}\left(\mathrm{H}_{2} \mathrm{PO}_{2}\right)_{6}\right]_{\left(\mathrm{H}_{2} \mathrm{PO}_{2}\right)_{2}}^{\frac{1 / 2}{\mathrm{SO}_{4}}}+4 \mathrm{H}_{2} \mathrm{O}(808.09)
$$

1. Salz mit $4 \mathrm{H}_{2} \mathrm{O}$.

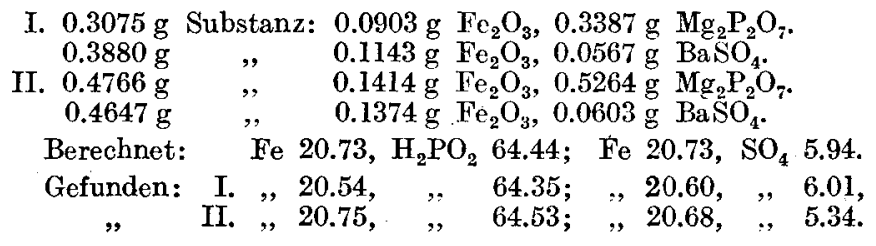

2. Wasserfreies Salze.

$0.1538 \mathrm{~g}$ Substanz: $0.0506 \mathrm{~g} \mathrm{Fe}_{2} \mathrm{O}_{3}, 0.1826 \mathrm{~g} \mathrm{Mg}_{2} \mathrm{P}_{2} \mathrm{O}_{7}$, $0.1630 \mathrm{~g} \quad, \quad 0.0533 \mathrm{~g} \mathrm{Fe}_{2} \mathrm{O}_{3}, 0.0237 \mathrm{~g} \mathrm{BaSO}_{4}$.

Berechnet: $\mathrm{Fe} 22.76, \mathrm{H}_{2} \mathrm{PO}_{2} 70.72 ; \mathrm{Fe} 20.76, \mathrm{SO}_{4} 6.52$.

Gefunden: „2 23.01, " 69.35; Fe 22.87, „5.98.

\section{Saures Sulfat.}

Man arbeitet möglichst konzentriert, indem man $2 \mathrm{~g}$ des Ausgangshypophosphits in $6 \mathrm{ccm}$ einer Schwefelsäure löst, die aus gleichen Volumen konzentrierter Säure und Wasser bereitet wurde. Dies dauert einige Zeit, da man nur ganz gelinde erwärmen darf. $\mathrm{Zu}$ dieser Lösung, die zuerst gelb, später aber (nach dem Erkalten) eigentümlich blaßrot gefärbt ist, fügt man zuerst noch wenige (3-4) Tropfen konzentrierter Schwefelsäure, dann langsam mehrere ccm absoluten Alkohol, wodurch die Verbindung als weißes Pulver gefällt wird. ${ }^{1}$ )

Man trennt von der Mutterlauge durch Aufstreichen auf Ton und wäscht mit wenig Alkohol nach. Man muß dann den Körper sogleich analysieren, da er die wiederholt erwähnte Umwandlung in die zähe Masse ziemlich rasch erleidet. Wir haben daher keine Gewähr dafür, daß er schon völlig trocken war. Deshalb läßt sich sein Wassergehalt nicht mit Sicherheit bestimmen.

$$
\left[\mathrm{Fe}_{3}\left(\mathrm{H}_{2} \mathrm{PO}_{2}\right)_{6}\right]\left(\mathrm{SO}_{4} \mathrm{H}\right)_{3}+\mathrm{xH}_{2} \mathrm{O} \text {. }
$$

I. $0.2834 \mathrm{~g}$ Substanz: $0.0602 \mathrm{~g} \mathrm{Fe}_{2} \mathrm{O}_{3}=14.83 \% \mathrm{Fe}$ und $0.1735 \mathrm{~g}_{\mathrm{Mg}_{2}} \mathrm{P}_{2} \mathrm{O}_{7}$ $=35.77 \% \mathrm{H}_{2} \mathrm{PO}_{2} ; 0.3457 \mathrm{~g}$ Substanz: $0.0728 \mathrm{~g} \quad \mathrm{Fe}_{2} \mathrm{O}_{3}=14.73 \% \mathrm{Fe}$ und $0.2291 \mathrm{~g} \mathrm{BaSO}=27.27 \% \mathrm{SO}_{4}$.

1) Da es immerhin möglich gewesen wäre, daß aus diesen Lösungen durch Alkohol Ferrisulfat gefällt wird, haben wir uns durch besondere Versuche davon überzeugt, daß aus Lösungen von Ferrisulfat in Schwefelsäure derselben Kon. zentration Alkohol nichts abscheidet. 
II. $0.3522 \mathrm{~g}$ Substanz: $0.0637 \mathrm{~g} \mathrm{Fe}_{2} \mathrm{O}_{3}=12.65 \%$ Fe und $0.1792 \mathrm{~g} \mathrm{Mg}_{2} \mathrm{P}_{2} \mathrm{O}_{7}$ $=29.73 \% \mathrm{H}_{2} \mathrm{PO}_{2} ; 0.3514 \mathrm{~g}$ Substanz: $0.0693 \mathrm{~g} \mathrm{Fe}_{2} \mathrm{O}_{3}=13.79 \% \mathrm{Fe}$ und $0.2128 \mathrm{~g} \mathrm{BaSO}_{4}=24.92 \% \mathrm{SO}_{4}{ }^{1}$ )

Hiernach verhalten sich $\mathrm{Fe}: \mathrm{H}_{2} \mathrm{PO}_{2} ; \mathrm{Fe}: \mathrm{SO}_{4}$.

$$
\begin{aligned}
\text { I. } 1: 2.07 ; & 1: 1.07 \text {, } \\
\text { II. } 1: 2.02 ; & 1: 1.05 \text {. }
\end{aligned}
$$

\section{Trisulfat-dihypophosphit.}

Man verfährt genau wie bei der Darstellunr des vorhergehenden Salze (Nr. 3, aber ohne nochmaligen Zusatz von konzentrier ier Schwefelsäure oder auch wie bei Nr. 2), fügt aber statt Alkohol tropfenweise Wasser hinzu. Hierbei scheidet sich die Verbindung als weißes Pulver ab, das auf Ton vorzüglich abtrocknet. Auch dieses Salz wandelt sich in die amorphe Masse um, aber viel langsamer als das vorhergehende, so daß es möglich ist, den Wassergehalt zu bestimmen, da man das Salz immerhin $1 / 2-1$ Stunde an der Luft auf Ton trocknen lassen kann

$$
\begin{gathered}
\mathrm{SO}_{4} \mathrm{H} \\
{\left[\mathrm{Fe}_{3}\left(\mathrm{H}_{2} \mathrm{PO}_{2}\right)_{6}\right] \mathrm{H}_{2} \mathrm{PO}_{2}} \\
\mathrm{SO}_{4} \mathrm{SO}_{4}
\end{gathered}+24 \mathrm{H}_{2} \mathrm{O} \text { (1968.5). }
$$

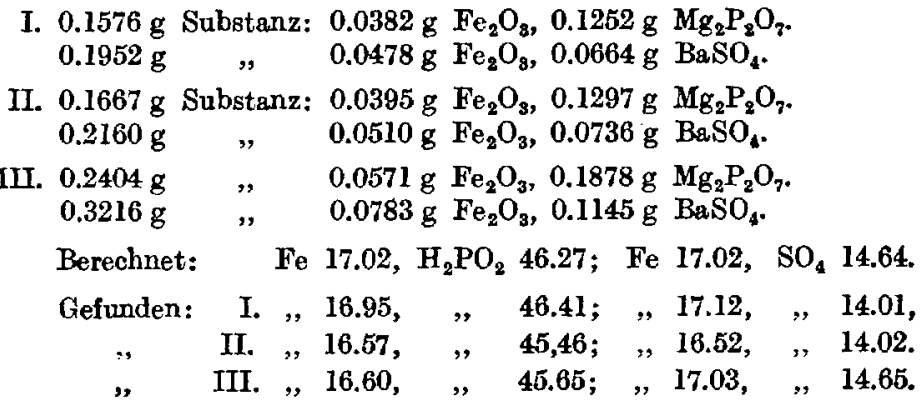

5. 4,5-Sulfat-monohypophosphit.

Man löst unter denselben Vorsichtsmaßregeln wie oben $2 \mathrm{~g}$ des Ausgangshypophosphits in $6 \mathrm{ccm}$ einer Schwefelsäure, die aus 1 Vol. konzentrierter Säure und $1^{1} / 2$ Vol. Wasser erhalten wurde, und fügt langsam einige ccm absoluten Alkohol hinzu. Man bekommt dieselbe Verbindung, wenn man auch etwas konzentriertere Schwefelsäure und gewöhnlichen Alkohol nimmt. Das Salz verhält sich im übrigen genau wie das vorhergehende und man verfährt bei der Isolierung ebenso wie bei ihm.

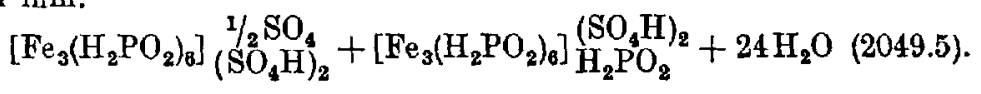

1) Wegen des verschiedenen Wassergehaltes sind die absoluten Werte natïrlich schwankend. 


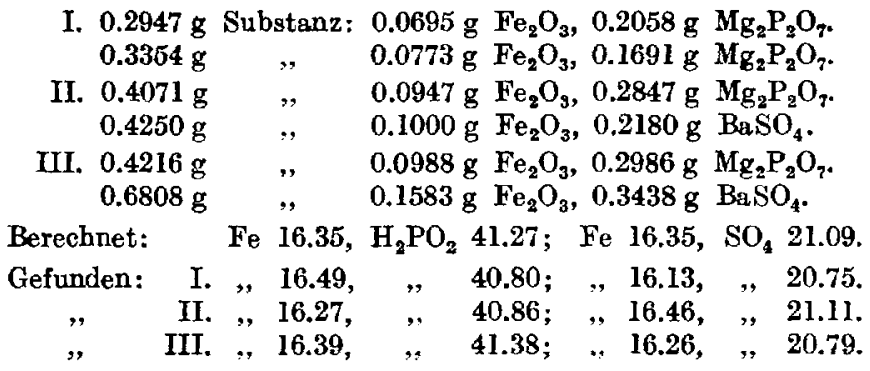

\section{Perchlorat, Hexachloroantimoniat, Salze mit Eisenchloro-(bromo-) Anionen.}

6. Diperchlorat-hypophosphit (S. 17).

Man löst etwa $2 \mathrm{~g}$ Ausgangshypophosphit in $20-25 \mathrm{ccm} 20 \%$ iger Uberchlorsäure unter Erwärmen. Beim Erkalten kristallisiert das Salz

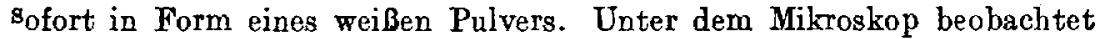
man traubige Aggregate und vereinzelt Blättchen von rhombischem Umriß und Stäbchen. Sogleich nach der Auskristallisation trennt man durch Aufstreichen auf Ton von der Mutterlauge und läßt wenige Stunden an der Luft trocknen, worauf man analysieren $\mathrm{muB}$, da bei längerem Liegen die Umwandlung in die amorphe, deutlich blaßrote Masse eintritt.

Man kann das Salz auch auf folgende Weise darstellen: zu der obigen Lösung_von Ferrihypophosphit in der Uberchlorsäure fügt man langsam und unter vorsichtigem Erwärmen noch weitere Mengen Ferrihypophosphit hinzu, solange Lösung eintritt. Man erhält eine eigentümlich blaßrote, etwas schwer flüssige Lösung, aus der nicht durch Kristallisation, sondern durch Zusatz von wenigen Tropfen Wasser oder auch Alkohol das Perchlorat derselben Zusammensetzung erhalten wird. (Große Vorsicht bei dieser Darstellung wegen der Explosionsgefahr!) Uber die explosiven Eigenschaften dieser Verbindung, besonders wenn sie entwässert ist ${ }_{\star}$ s. oben S. 25. Das lufttrockene Salz enthält 18 Moleküle Wasser.

$$
\left[\mathrm{Fe}_{3}\left(\mathrm{H}_{2} \mathrm{PO}_{2}\right)_{6}\right] \mathrm{H}_{2} \mathrm{PO}_{2}+1 / 2 \mathrm{H}_{3} \mathrm{PO}_{2}+18 \mathrm{H}_{2} \mathrm{O}(1179.18) \text {. }
$$

I. $0.2932 \mathrm{~g}$ Substanz: $0.0602 \mathrm{~g} \mathrm{Fe}_{2} \mathrm{O}_{3}, 0.2070 \mathrm{~g} \mathrm{Mg}_{2} \mathrm{P}_{2} \mathrm{O}_{7}$;

$$
0.4685 \mathrm{~g}, \quad 0.0911 \mathrm{~g} \mathrm{AgCl} .^{1} \text { ) }
$$

1) Zur Bestimmung der Utberchlorsäure in dieser explosiven Verbindung kann man nicht einfach, wie üblich, mit wasserfreiem Natriumcarbonat erhitzen, sondern man $m u B$ die unterphosphorige Säure vorher oxydieren. Hierzu löst man im Platintiegel in wenig konzentrierter Salpeteraäure langsam auf, wobei bald unter lebhafter Entwicklung von nitrosen Gasen Oxydation eintritt. Man fügt dann überschüssiges Natriumcarbonat hinzu, verdampft zur Trockene, glüht mäBig usw. - Man kann aber auch in wenig verdünnter Schwefelsäure lösen und mit Kaliumpermanganat oxydieren, wo bei sich Mangandioxyd abscheidet. Hierauf wird wiederum überschüssiges Natriumcarbonat hinzugefügt usw. 


\begin{tabular}{|c|c|c|c|c|c|c|c|c|}
\hline II. & $\begin{array}{l}0.1518 \mathrm{~g} \\
0.2182 \mathrm{~g}\end{array}$ & & & $\begin{array}{l}0.0286 \mathrm{~g} \\
0.0514 \mathrm{~g}\end{array}$ & $\begin{array}{l}\mathrm{Fe}_{2} \mathrm{O}_{3}, \\
\mathrm{AgCl} \text {. }\end{array}$ & $0.1086 \mathrm{~g}$ & $\mathrm{Mg}$ & $\mathrm{O}_{7}$ \\
\hline Ir. & $\begin{array}{l}0.1343 \mathrm{~g} \\
0.1904 \mathrm{~g}\end{array}$ & ", & & $\begin{array}{l}0.0270 \mathrm{~g} \\
0.0442 \mathrm{~g}\end{array}$ & $\begin{array}{l}\mathrm{Fe}_{2} \mathrm{O}_{3}, \\
\mathrm{AgCl} .\end{array}$ & $0.0943 \mathrm{~g}$ & $\mathrm{Mg}$ & $\mathrm{o}_{7}$ \\
\hline IV. & $\begin{array}{l}0.2122 \mathrm{~g} \\
0.1957 \mathrm{~g}\end{array}$ & ,", & & $\begin{array}{l}0.0400 \mathrm{~g} \\
0.0480 \mathrm{~g}\end{array}$ & $\begin{array}{l}\mathrm{Fe}_{2} \mathrm{O}_{3}, \\
\mathrm{AgCl} \text {. }\end{array}$ & g & & $\partial_{7} ;$ \\
\hline V. & $\begin{array}{l}0.1710 \mathrm{~g} \\
0.1101 \mathrm{~g}\end{array}$ & ", & & $\begin{array}{l}0.0348 \mathrm{~g} \\
0.0450 \mathrm{~g}\end{array}$ & $\begin{array}{l}\mathrm{Fe}_{2} \mathrm{O}_{3}, \\
\mathrm{AgCl} \text {. }\end{array}$ & & & \\
\hline \multicolumn{3}{|c|}{ Berechnet: } & & Fe 14.21 & $\mathrm{H}_{2} \mathrm{~F}$ & $\partial_{2} 41.34$ & $\mathrm{ClO}_{4}$ & 16.87. \\
\hline & Gefv & & $\begin{array}{l}\text { I. } \\
\text { III. } \\
\text { III. } \\
\text { V. }\end{array}$ & $\begin{array}{ll}, & 14.36 \\
, " & 14.50 \\
, " & 14.06 \\
, " & 14.52 \\
,, & 14.23\end{array}$ & ", & $\begin{array}{l}41.25 \\
41.80 \\
41.03 \\
42.00 \\
41.82\end{array}$ & $\begin{array}{l}\text { ", } \\
\text { ", } \\
\text { " }\end{array}$ & $\begin{array}{l}17.05 . \\
16.35 . \\
16.10 . \\
17.02 . \\
16.98 .\end{array}$ \\
\hline
\end{tabular}

7. Hexachloroantimoniat-Hypophosphit (S. 18).

Man löst $21 / 2 \mathrm{~g}$ Ferrihypophosphit in $8 \mathrm{ccm}$ konzentrierter Salzsäure (etwa $38 \%$ ig) ohne Erwärmen auf und fügt $8 \mathrm{~g}$ feste HexachloroantimonBäure (s. Anm. 2, \$. 16) hinzu. Es bildet sich sofort ein Niederschlag, dessen Menge sich vermehrt, während sich die Hexachlorantimonsäure löst. Absaugen, Trocknen auf Ton. Sehr fein kristallinisches, blaßgelbes Pulver. Enthält lufttrocken 18 Moleküle Wasser, die über Chlorcalcium sehr bald völlig sich verflüchtigen. Diese Verbindung behält dauernd ihre feinpulvrige Beschaffenheit.

$$
\left[\mathrm{Fe}_{3}\left(\mathrm{H}_{2} \mathrm{PO}_{2}\right)_{6}\right] \underset{\mathrm{H}_{2} \mathrm{PO}_{2}}{\left(\mathrm{SbCl}_{6}\right)_{2}}+\left[\mathrm{Fe}_{3}\left(\mathrm{H}_{2} \mathrm{PO}_{2}\right)_{6}\right]_{\left(\mathrm{H}_{2} \mathrm{PO}_{2}\right)_{2}}^{\mathrm{SbCl}_{6}}+18 \mathrm{H}_{2} \mathrm{O}(2634.1) \text {. }
$$

I. $0.2516 \mathrm{~g}$ Substanz: $0.0460 \mathrm{~g} \quad \mathrm{Fe}_{2} \mathrm{O}_{3}, 0.1602 \mathrm{~g} \quad \mathrm{Mg}_{2} \mathrm{P}_{2} \mathrm{O}_{7}$ und $0.0638 \mathrm{~g}$ $\mathrm{Sb}_{2} \mathrm{~S}_{3}+\mathrm{S}$, wovon $0.0634 \mathrm{~g}$ (im $\mathrm{CO}_{2}$-Strom erhitzt) $0.0480 \mathrm{~g} \mathrm{Sb}_{2} \mathrm{~S}_{3}$ lieferten ${ }^{1}$ ); $0.2603 \mathrm{~g}$ Substanz: $0.0475 \mathrm{~g} \mathrm{Fe}_{2} \mathrm{O}_{3}, 0.0576 \mathrm{~g} \quad \mathrm{Sb}_{\mathrm{z}} \mathrm{S}_{3}+\mathrm{S}$, wovon $0.0562 \mathrm{~g} 0.0498 \mathrm{~g} \mathrm{Sb}_{2} \mathrm{~S}_{3} ; 0.1560 \mathrm{~g}$ Substanz: $0.1533 \mathrm{~g} \mathrm{AgCl}$.')

II. $0.3900 \mathrm{~g}$ Substanz: $0.0708 \mathrm{~g} \mathrm{Fe}_{2} \mathrm{O}_{3}, 0.2470 \mathrm{~g} \mathrm{Mg}_{2} \mathrm{P}_{2} \mathrm{O}_{7}, 0.2214 \mathrm{~g} \mathrm{Sb}_{2} \mathrm{~S}_{3}+\mathrm{S}$, wovon $0.1852 \mathrm{~g} 0.0631 \mathrm{~g} \quad \mathrm{Sb}_{2} \mathrm{~S}_{3} ; 0.2050 \mathrm{~g}$ Substanz: $0.0373 \mathrm{~g}^{\mathrm{F}} \mathrm{Fe}_{2} \mathrm{O}_{3}$, $0.0473 \mathrm{~g} \mathrm{Sb}_{2} \mathrm{~S}_{3}+\mathrm{S}$, wovon $0.0467 \mathrm{~g} 0.0392 \mathrm{~g} \mathrm{Sb}_{2} \mathrm{~S}_{3} ; 0.2464 \mathrm{~g}$ Substanz: $0.2428 \mathrm{~g} \mathrm{AgCl}$.

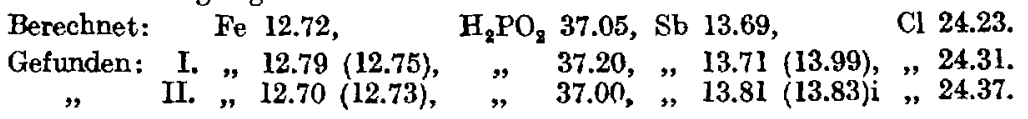

8. Trichloro-hy pophosphitoferriat-Hy pophosphit (vgl.oben \$.18).

Man übergießt $5 \mathrm{~g}$ Ausgangshypophosphit mit $10 \mathrm{ccm}$ konzentrierter Salzsäure (von etwa $38 \%$ ) und läßt bei gewöhnlicher Temperatur stehen.

1) Das Antimon wurde in salzsaurer Lösung der Substanz mit Schwefelwasserstoff gefällt oder man oxydiert zunächst, wie immer, mit Brom in salzsaurer Lösung, fügt Ammoniak und Schpefelammon im ÜberschuB hingr, filtriert von abgeschiedenem Schwefeleisen ab und fällt Antimonsulfid aus der Sulfosalzlösung mit verdünnter Schwefelsäure.

ग) Aus der mit Hilfe von otwa $6 \mathrm{~g}$ Weinsäure bereitoten, mit Salpetersäure versetzten Lösung abgeschieden. 
Hierbei bemerkt man bald den Beginn der Umwandlung des Hypophosphits in ein gelbes, kristallisiertes Pulver, welche nach spätesten 2 Tagen vollendet ist. Die Verbindung, die man als leitendes Salz der Reihe benützen kann, hat ein großes Bildungsbestreben, man erbält sie aus beliebigen anderen Hypophosphiten mit konzentrierter Salzsäure. Von dieser kann man auch mehr oder etwas weniger als oben angegeben nehmen. Unter dem Mikroskop vorzüglich ausgebildete, durchsichtige, gelbe Oktaeder mit häufig abgeschnittenen Ecken. Größere, mit bloßem Auge sichtbare Oktaeder bekommt man durch vorsichtiges Umkristallisieren aus konzentrierter, mäßig erwärmter Salž äure oder auch indem man bei der Darstellung von vornherein mehr Salzsäure nimmt. Absaugen, Trocknen auf Ton. Lufttrocken besitzt es 12 Moleküle Wasser. Diese verflüchtigen sich leicht über Chlorcalcium. Das Salz behält seine kristallinische Beschaffenheit dauernd bej.

$$
\left[\mathrm{Fe}_{3}\left(\mathrm{H}_{2} \mathrm{PO}_{2}\right)_{6}\right]\left[\begin{array}{l}
\mathrm{Fe}_{\mathrm{H}_{2}}^{\mathrm{H}_{2} \mathrm{PO}_{2}} \\
\mathrm{PO}_{2}
\end{array}\right]_{2}+12 \mathrm{H}_{2} \mathrm{O}(1293.69)
$$

1. Salz mit 12 Molekülen Wasser.

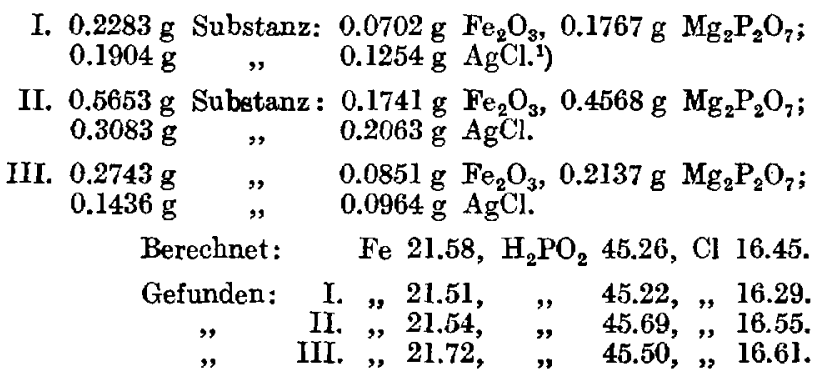

2. Wasserfreies Salz.
I. $0.3123 \mathrm{~g}$ Substanz: $0.1158 \mathrm{~g} \mathrm{Fe}_{2} \mathrm{O}_{3}, 0.2903 \mathrm{~g} \mathrm{Mg} \mathrm{P}_{2} \mathrm{O}_{7}$; $0.2950 \mathrm{~g} \quad, \quad 0.2359 \mathrm{~g} \mathrm{AgCl}$.
II. $0.2840 \mathrm{~g}, \quad 0.1048 \mathrm{~g} \mathrm{Fe}_{2} \mathrm{O}_{3}, 0.263 \mathrm{~g} \mathrm{Mg} \mathrm{Mg}_{2} \mathrm{P}_{2} \mathrm{O}_{7}$; $0.2532 \mathrm{~g} \quad, \quad 0.1997 \mathrm{~g} \mathrm{AgCl}$.
Berechnet: $\quad \mathrm{Fe} 25.91, \mathrm{H}_{2} \mathrm{PO}_{2}$ 54.34, $\mathrm{Cl} 19.75$.
Gefunden: I. , 25.93, , $54.31,, 19.78$. " II. ", 25.81, " $, 54.12, " 19.51$.

9. Tetrachloroferriat-Dihypophosphit (S. 18).

Aus der gelbbraunen Mutterlauge der vorhergehenden oktaedrischen Verbindung scheiden sich über Schwefelsäure zunächst meistens noch

1) Um eine Reduktion des Silbers durch die unterphosphorige Säure zu vermeiden, fällt man in verdünnter salpetersaurer Lð̌sung ohne zu erwärmen. Tritt trotzdem Reduktion ein, so löst man das erstmals erhaltene Silberchlorid in Ammoniak, filtriert usw. 
einige Oktaeder aus, und hierauf blaßgelbe Blättchen, die unter dem Mikroskop sehr deutlich vierseitige, rechtwinklige Tafeln mit teilweise abgeschnittenen Ecken darstellen. Es ist aber zu bemerken, daß man nicht jedes Mal aus der Mutterlauge des oktaedrischen Körpers die Verbindung bekommt, und wir sind nicht imstande die genaueren Bedingungen, unter denen man sie mit Sicherheit erhält, anżugeben. Scheidet sich die Verbindung überhaupt aus, dann geschieht das ziemlich rasch nach den Oktaedern, bei längerem Stehen bildet sich schließlich so viel Ferrochlorid, daß dieses auskristallisiert.

Man streicht auf Ton und analysiert nach etwa einer Stunde. LäBt man dièse übersaure Verbindung länger liegen, so verwandelt sie sich unter Abgabe von Salzsäure in eine gelbe zusammenklebende Masse.

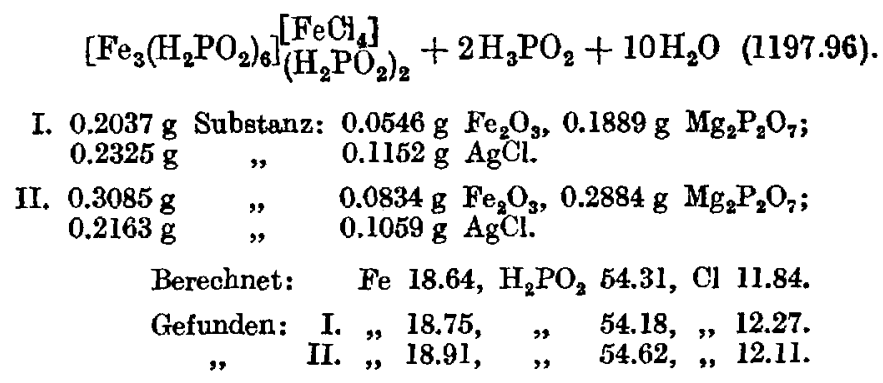

(Ein weiteres Salz einer Eisenchlorosäure A. S. 35.)

10. Tetrabromoferriat-Dihypophosphit (S. 18).

Man übergieBt $5 \mathrm{~g}$ Ausgangshypophosphit mit etwa $10 \mathrm{ccm}$ rauchender (bromfreier) Bromwasserstoffsäure (von etwa $50 \%$ ). Schon nach kurzer Zeit beginnt die Umwandlung und ist im Laufe von 1-2 Tagen vollendet. Absaugen, trocknen auf Ton. Dunkelrotbraunes, kleinkristallinisches Pulver; unter dem Mikroskop größere und kleinere Blättchen von unregelmäßigem Umriß, schon in sehr dünner Schicht deutlich rotbrann. Nach 2-3 stündigem Liegen an der Luft enthält es 12 Moleküle Wasser. Es behält seine kristallinische Beschaffenheit dauernd bei.

$$
\left[\mathrm{Fe}_{3}\left(\mathrm{H}_{2} \mathrm{PO}_{2}\right)_{8}\right]_{\left(\mathrm{H}_{2} \mathrm{PO}_{2}\right)_{2}}^{[\mathrm{FeBr}]}+12 \mathrm{H}_{2} \mathrm{O}(1279.71)
$$

I. $0.3581 \mathrm{~g}$ Substanz: $0.0883 \mathrm{~g} \mathrm{Fe}_{2} \mathrm{O}_{3}, 0.2447 \mathrm{~g} \mathrm{Mg}_{2} \mathrm{P}_{2} \mathrm{O}_{3}$;

$0.1306 \mathrm{~g} \quad 0.0767 \mathrm{~g} \mathrm{AgBr}$.

II. $\begin{array}{lll}0.3528 \mathrm{~g} & \Rightarrow & 0.0874 \mathrm{~g} \mathrm{Fe} \mathrm{F}_{2}, 0.2450 \mathrm{~g} \mathrm{Mg}_{2} \mathrm{P}_{2} \mathrm{O}_{2} ; \\ 0.2350 \mathrm{~g} & \Rightarrow & 0.1378 \mathrm{~g} \mathrm{AgBr} .\end{array}$

Berechnet: $\quad \mathrm{Fe} 17.45, \mathrm{H}_{2} \mathrm{PO}_{2} 40.67, \mathrm{Br} 24.98$.

Gefunden: I. "17.25, , $39.83, \quad$,24.99. " II. " 17.33, ", 40.57, ", 24.95.

Z. anorg. u. allg. Chem. Bd. 106. 


\section{Verbindangen, welche man aus Natriumbypophosphit und Ferrisalzen erhält.}

(Vgl. oben S. 16.)

Diese Verbindungen erhält man, wenn man auf Ferrisalze weniger als die äquivalente Menge Natriumhypophosphit einwirken läßt. Bei äquivalenter Menge bekommt man die reinen Hypophosphite, wie das Ausgangshypophosphit und die weiter unten zu beschreibenden Salze Nr. 18 bis 22. Die Verbindungen enthalten, mit Ausnahme einer einzigen (Nr. 14), Hydroxogruppen. Sie stellen weißliche Pulver dar, trocknen auf Ton gut ab und erleiden die Umwandlung in die zusammenklebende Masse nicht.

\section{Disulfat-hypophosphit.}

Man löst $5.0 \mathrm{~g}$ Ferrisulfat $\left(\mathrm{Fe}_{2}\left(\mathrm{SO}_{4}\right)_{3} .6 \mathrm{H} \mathrm{O}, 1 / 100 \mathrm{Mol}\right)$ und $4.2 \mathrm{~g}$ $\mathrm{NaH}_{2} \mathrm{PO}_{2} \cdot \mathrm{H}_{2} \mathrm{O}(4 / 100 \mathrm{Mole})$ je in etwa $15 \mathrm{ccm}$ Wasser, vermischt beide Lösungen und erwärmt. Hierbei scheidet sich die Verbirdung in Form eines feinen, weißlichen Pulvers aus. Arbeitet man in wesentlich größarer Verdünnung, so bekommt man immer schwefelsäureärmere Salze und schließlich ein reines Hypophosphit, das sich dem Verhältnis 3:8.5 annähert (vgl. Nr. 18). Auf Ton getrocknet enthält es nach 1 bis 2 Stunden 24 Moleküle Wasser. Diese verflüchtigen sich über Chlorcalcium sämtlich ziemlich rasch.

$$
\begin{aligned}
& {\left[\mathrm{Fe}_{3}\left(\mathrm{H}_{2} \mathrm{PO}_{2}\right)_{6}\right] \mathrm{H}_{2} \mathrm{SO}_{4}+\left[\mathrm{Fe}_{3} \underset{(\mathrm{OH})}{\left(\mathrm{H}_{2} \mathrm{PO}_{2}\right)_{6}}\right] \mathrm{SO}_{4}+24 \mathrm{H}_{2} \mathrm{O}(1822.33) \text {. }} \\
& \text { I. } 0.2032 \mathrm{~g} \text { Substanz: } 0.0535 \mathrm{~g} \mathrm{Fe}_{2} \mathrm{O}_{3}, 0.1623 \mathrm{~g} \mathrm{Mg}_{2} \mathrm{P}_{2} \mathrm{O}_{7} \text {. } \\
& 0.3204 \mathrm{~g} \quad, \quad 0.0834 \mathrm{~g} \mathrm{Fe}_{2} \mathrm{O}_{3}, 0.0809 \mathrm{~g} \mathrm{BaSO}^{\text {. }} \\
& \text { II. } 0.1073 \mathrm{~g} \quad " \quad 0.0290 \mathrm{~g} \mathrm{Fe}_{2} \mathrm{O}_{3}, 0.0860 \mathrm{~g} \mathrm{Mg}_{3} \mathrm{P}_{2} \mathrm{O}_{7} \text {. } \\
& 0.2172 \mathrm{~g} \quad " \quad 0.0590 \mathrm{~g} \mathrm{Fe}_{2} \mathrm{O}_{3}, 0.0570 \mathrm{~g} \mathrm{BaSO} \text {. }
\end{aligned}
$$

12. Dichlorid-monohypophosphit.

Man löst $5.4 \mathrm{~g} \mathrm{FeCl}_{3} \cdot 6 \mathrm{H}_{2} \mathrm{O}(2 / 100 \mathrm{Mole})$ und $4.2 \mathrm{~g} \mathrm{NaH}_{2} \mathrm{PO}_{2} \cdot \mathrm{H}_{2} \mathrm{O}$ ( $4 / 100$ Mole) je in etwa $20 \mathrm{ccm}$ Wasser, vereinigt beide Lösungen und erwärmt. Hierbei trübt sich die Flüssigkeit und beim Erkalten scheidet sich die Verbindung als lockeres weiBliches Pulver ab. Bei gröBerer Verdünnung der Lösungen bekommt man immer chlorärmere Fällungen (vgl, Nr. 11) und schließlich ein chlorfreies Hypophosphit anrähernd vom Verbältnis 3:8, wie es das Ausgangshypophosphit zeigt. Arbeitet man andererseits so konzentriert wie irgend möglich nnd mit weniger Natriumhypophosphit so erhält man das Chloroferriat Nr. 14. Immerhin erhält man das in Rede stehende Chlorid bei ziemlich erheblichen Schwan- 
kungen der Wassermenge. Im übrigen verfährt man wie bei den vorhergehenden Salzen.

$$
\begin{aligned}
& {\left[\mathrm{Fe}_{3}\left(\mathrm{H}_{2} \mathrm{PO}_{2}\right)_{6}\right] \mathrm{Cl}_{2}+\left[\mathrm{Fe}_{3} \underset{(\mathrm{OH})_{2}}{\left(\mathrm{H}_{2} \mathrm{PO}_{3}\right)_{6}}\right] \mathrm{H}_{2} \mathrm{PO}_{2}+15 \mathrm{H}_{2} \mathrm{O}(1573.00) .} \\
& \text { I. } 0.1602 \mathrm{~g} \text { Substanz: } 0.0489 \mathrm{~g} \mathrm{Fe} \mathrm{O}_{2}, 0.1474 \mathrm{~g} \mathrm{Mg}_{2} \mathrm{P}_{2} \mathrm{O}_{7} \text {; }
\end{aligned}
$$

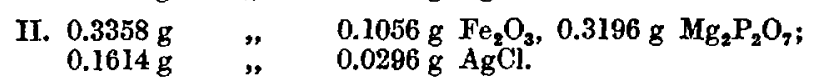

$$
\begin{aligned}
& \text { III. } 0.1876 \mathrm{~g} \quad \text { " } \quad 0.0570 \mathrm{~g} \mathrm{Fe}_{2} \mathrm{O}_{3}, 0.1740 \mathrm{~g} \mathrm{Mg}_{2} \mathrm{P}_{2} \mathrm{O}_{2} \text {; } \\
& 0.1080 \mathrm{~g} \quad \ddot{0} \quad 0.0206 \mathrm{~g} \mathrm{AgCl} \text {. } \\
& \text { Berechnet: } \quad \mathrm{Fe} 21.30, \mathrm{H}_{2} \mathrm{PO}_{2} \text { 53.77, } \mathrm{Cl} 4.51 \text {. } \\
& \text { Gefunden: I. " 21.35, ” } 53.82, " 4.52 \text {. } \\
& \text { " III. " } 22.00, \quad 21.25, \quad \text { " } 55.61, ", 4.54 \text {. }
\end{aligned}
$$

13. Dinitrat-trihypophosphit.

Man löst $8.0 \mathrm{~g}$ Ferrinitrat $\left(\mathrm{Fe}\left(\mathrm{NO}_{3}\right)_{3} .9 \mathrm{H}_{2} \mathrm{O}, 2 / 100\right.$ Mole) und $4.2 \mathrm{~g}$ Natriumhypophosphit ( $4 / 100$ Mole) je in etwa $20 \mathrm{ccm}$ Wasser, vereinigt beide Lösungen und erwärmt. Für alles übrige gilt das im vorhergehenden Gisagte.

$$
\begin{aligned}
& \left.\left[\mathrm{Fe}_{3}\left(\mathrm{H}_{2} \mathrm{PO}_{2}\right)_{6}\right] \underset{\left(\mathrm{H}_{2} \mathrm{PO}_{2}\right)_{2}}{\stackrel{\mathrm{NO}_{3}}{\mathrm{H}_{3}}+\left[\mathrm{Fe}_{3}\right.} \stackrel{\left(\mathrm{H}{ }_{2}{ }^{*} \mathrm{O}_{2}\right)_{8}}{(\mathrm{OH})}\right] \underset{\mathrm{H}_{2} \mathrm{PO}_{2}}{\mathrm{NO}_{3}}+24 \mathrm{H}_{2} \mathrm{O} \\
& \text { I. } 0.3387 \mathrm{~g} \text { Substanz: } 0.0859 \mathrm{~g} \mathrm{Fe}_{2} \mathrm{O}_{3} \text {, } 0.3019 \mathrm{~g} \mathrm{Mg}_{2} \mathrm{P}_{2} \mathrm{O}_{7} \text {; } \\
& \left.0.4538 \mathrm{~g} \quad, \quad 4.8 \mathrm{~cm} 1 / 10 \mathrm{n} \cdot \mathrm{HCl} .{ }^{1}\right)
\end{aligned}
$$

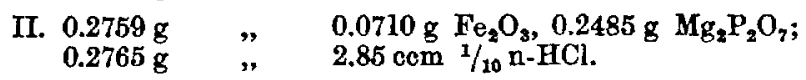

$$
\begin{aligned}
& \text { III. } 0.1108 \mathrm{~g} \quad, \quad 0.0274 \mathrm{~g} \mathrm{Fe}_{2} \mathrm{O}_{3}, 0.0948 \mathrm{~g} \mathrm{Mg}_{2} \mathrm{P}_{2} \mathrm{O}_{7} \text {; } \\
& 0.3620 \mathrm{~g} \quad \# \quad 3.55 \mathrm{ccm} 1 / 10 \mathrm{n} \cdot \mathrm{HCl} \text {. } \\
& \text { Berechnet: } \quad \mathrm{Fe} \text { 17.78, } \mathrm{H}_{2} \mathrm{PO}_{2} 61.79, \mathrm{NO}_{8} 6.58 \text {. } \\
& \text { Gefunden: I. , 17.74, ” 52.07, \# 6.56. } \\
& \text { " II. " } 18.00, \quad " \quad 52.62, \quad " \quad 6.39 \text {. }
\end{aligned}
$$

14. Tetrachloroferriat-trichlorohy pophosphitoferriathypophosphit (S. 18).

Bei der Darstellung dieses Salzes muß man möglichst konzentriert arbeiten. Man löst $5.4 \mathrm{~g} \mathrm{FeCl} 3.6 \mathrm{H}_{2} \mathrm{O}\left({ }^{2} / 100\right.$ Mole) in sehr wenig $(2 \mathrm{ccm})$ Wasser tnd fügt hierzu unter Erwärmen und Umrübren allmählich eine konzentrierte Lösung von $2 / 100$ Molen $\mathrm{NaH}_{2} \mathrm{PO}_{2} \cdot \mathrm{H}_{2} \mathrm{O}(2.2 \mathrm{~g}$ in $2 \mathrm{ccm}$ Wasser). Der zunächst entstehende gelbe, in der Hitze zähe Niederschlag löst sich jedes Mal wieder auf, bis auf einmal in der ganzen Flüssigkeit ein gelbe. 3 kristallinisches Pulver sich abscheidet. Man saugt noch heiB sogleich $a b$, um eine möglichst kleine Beimengung von Natriumchlorid

1) B.stimmung der Salpetersäure durch Reduktion za Ammoniak in alkalischer LLösung mit Devarosscher Legierung. 
zu bekommen, streicht auf Ton und läßt einige Stunden trocknen. Gelbes kristallinisches Pulver, etwas dunkler gelb als das oktaedrische Salz Nr. 8 (S. 31), sonst diesem ganz gleich.

Zur Analyse nimmt man womöglich Präparate, welche bei mikroskopischer Betrachtung keine Würfel von Chlornatrium erkennen lassen. Andernfalls muß man eine Natriumbestimmung machen und das diesem entsprechende Chlor vom Gesamtchlor abziehen. Die Chlornatrium enthaltenden Präparate erscheinen heller gefärbt und färben die Bunsenflamme dauernd intensiv gelb.

$$
\left[\mathrm{Fe}_{3}\left(\mathrm{H}_{2} \mathrm{PO}_{2}\right)_{6}\right]\left[\begin{array}{c}
{\left[\mathrm{FeCl}_{4}\right]} \\
\mathrm{Fe}_{\mathrm{Cl}_{3}}^{\left.\mathrm{Cl}_{3} \mathrm{PO}_{2}\right)} \\
\mathrm{H}_{2} \mathrm{PO}_{2}
\end{array}\right]+6 \mathrm{H}_{2} \mathrm{O}(1156.00) .
$$

I. $0.1907 \mathrm{~g}$ Substanz: $0.0667 \mathrm{~g} \mathrm{Fe}_{2} \mathrm{O}_{3}, 0.1485 \mathrm{~g} \mathrm{Mg}_{8} \mathrm{P}_{2} \mathrm{O}_{7}$;

$$
0.1851 \mathrm{~g} " \quad 0.1612 \mathrm{~g} \mathrm{AgCl} \text {. }
$$

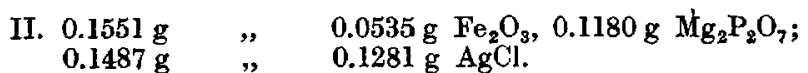

Berechnet: $\quad \mathrm{Fe} 24.15, \mathrm{H}_{2} \mathrm{PO}_{2} 45.02$, $\mathrm{Cl} 21.47$.

Gefunden: I. "24.10, \#45.48, ,21.54.

. " II. ", 24.14, " 44.46, ", 21.31.

Uber $\mathrm{CaCl}_{2}$ entwässertes Salz: $0.1871 \mathrm{~g}$ Substanz: $0.0732 \mathrm{~g} \mathrm{Fe}_{2} \mathrm{O}_{3}, 0.1624 \mathrm{~g}$ $\mathrm{Mg}_{2} \mathrm{P}_{2} \mathrm{O}_{2} ; 0.1270 \mathrm{~g}$ Substanz: $0.1232 \mathrm{~g}$ AgCl.

Berechnet: $\mathrm{Fe} 26.64, \mathrm{H}_{2} \mathrm{PO}_{2} 49.67, \mathrm{Cl} 23.69$.

Gefunden: $, 27.36, \quad$, 50.69, „24,00.

Chlornatriumhaltiges Präparat: 0.1546 g Substanz: $0.0524 \mathrm{~g} \mathrm{Fe}_{2} \mathrm{O}_{3}=23.72 \%$ Fe und $0.1170 \mathrm{~g} \mathrm{Mg}_{2} \mathrm{P}_{2} \mathrm{O}_{7}=44.21 \% \mathrm{H}_{2} \mathrm{PO}_{2} ; 0.0836 \mathrm{~g}$ Substanz: $0.0857 \mathrm{~g} \mathrm{AgCl}$ $=25.36 \% \mathrm{Cl} ; 1.3250 \mathrm{~g}$ Substanz: $0.0974 \mathrm{~g} \mathrm{Na} 2 \mathrm{SO}_{4}=2.38 \% \mathrm{Na}$, was entspricht $3.67 \% \mathrm{Cl}$.

Hiernach verhalten sich $\mathrm{Fe}: \mathrm{H}_{2} \mathrm{PO}_{2}: \mathrm{Cl}=1: 1.60: 1.44$.

\section{Verbindungen, welche bei der Einwirkung von onterphosphoriger Bãure auf Ferrisalze entstehen. \\ (Vgl. oben S. 19.)}

Wie bereits ausgeführt, bekommt man auf diese Weise keine einheitlichen Stoffe, aber aus ihrer Zusammensetzung geht immerhin soviel hervor, daB sie das Hexahypophosphito-triferri-Kation enthalten.

15. Perchlorat-hypophosphite.

Wir haben aus Ferriperchlorat und unterphosphoriger Säure 2 Reihen von Perchlorathypophosphiten dargestellt, und zwar die erste durch $\mathrm{Zu}$ satz der unterphosphorigen Säure zu Ferriperchloratlösung, die zweite umgekehrt. Wir verwendeten eine molekular-normale Ferriperchlorat- 
lösung $\left(462.3 \mathrm{~g} \mathrm{Fe}\left(\mathrm{ClO}_{4}\right)_{3} \cdot 6 \mathrm{H}_{2} \mathrm{O}^{1}\right)$ in $1 \mathrm{l}$ und eine $25 \%$ ige unterphosphorigo Säure.

\section{Reihe.}

Man erwärmt $10 \mathrm{ccm}$ der Ferriperchloratlösung und fügt tropfenweise die Lösung der unterphosphorigen Säure hinzu. Bei Zusatz jeden Tropfens tritt ein Niederschlag auf, der aber wieder verschwindet, bis man 2 bis 21/2 ccn zugesetzt hat. Hört man jetzt mit dem Zusatz auf, so kristallisiert sofort ein $\mathrm{Salz}$ aus, dessen Zusammensetzung sehr nahe beim Verhältnis $3 \mathrm{Fe}: 7 \mathrm{H}_{2} \mathrm{PO}_{2}: 2 \mathrm{ClO}_{4}$ liegt. Man hat bei dieser Darstellung eine gewisse Gewähr, stets dieses so zusammengesetzte $\mathrm{Salz}$ zu bekommen. Denn solange noch nicht so viel unterphosphorige Säure wie oben angegeben zugesetzt wurde, scheidet sich aus der Lösung nichts aus; hei längerem Stehen kristallisiert schließlich Ferroperchlorat $\left(\mathrm{Fe}\left(\mathrm{ClO}_{4}\right)_{2} \cdot 6 \mathrm{H}_{2} \mathrm{O}\right)$ aus. Wie man sieht, stimmt die Zusammensetzung des Salzes mit dem früher beschriebenen Diperchlorathypophosphit (Nr.6) überein, nur daB dieses noch $1 / 2$ Molekül freie unterphosphorige Säure enthält, während das vorliegende ein Neutralsalz ist:

$$
\left[\mathrm{Fe}_{3}\left(\mathrm{H}_{2} \mathrm{PO}_{2}\right)_{6}\right]_{\mathrm{H}_{2} \mathrm{PO}_{2}}^{\left(\mathrm{ClO}_{2}\right)_{2}}+\mathrm{xH}_{2} \mathrm{O} \text {. }
$$

In dem Maße, in dem man weiter unterphosphorige Säure hinzufügt, nimmt in den abgeschiedenen Verbindungen die Utberchlorsäure ab und die unterphosphorige Säure zu. Hiermit geht eine geringe Zunahme der Acidität parallel. Alle dargestellten Verbindungen liegen zwischen dem schon angegebenen Verhältnis

$$
3 \mathrm{Fe}: 7 \mathrm{H}_{2} \mathrm{PO}_{2}: 2 \mathrm{ClO}_{4} \text { und } 6 \mathrm{Fe}: 17 \mathrm{H}_{2} \mathrm{PO}_{2}: 3 \mathrm{ClO}_{4} \text {. }
$$

Diese Sálze sind kristallinisch, blaßrot und trocknen auf Ton vorzüglich ab. Lufttrocken enthalten sie alle Wasser, verwittern aber bald. Nicht selten bulden die Präparate, wohl auch infolge des Erbitzens der Lösung bei der Darstellung, zusammenhängende hautartige Gebilde; es macht den Eindruck, als wären die einzelnen Kriställehen verklebt.") Die Salze sind, wie das oben beschriebene Perchlorat, explosiv.

Die folgende Tabelle enthält die Reihe dieser Verbindungen, welche man durch Zusatz von 25\% iger unterphosphoriger Säure zu $10 \mathrm{ccm}$ molekular-normaler Ferriperchloratlösung bekommt

Angewandte Menge
$\mathrm{H}_{3} \mathrm{PO}_{2}(25 \% \mathrm{ig})$
a) (ast $2 \mathrm{ccm}$
b) slark $2 \mathrm{ccm}$
c)
d) etwa $3 \mathrm{ccm}$
e) etwa $5 \mathrm{ccm}$
f) etwa $6 \mathrm{ccm}$

\section{Zusammensetzung des erhaltenen Salzes}

$$
\mathrm{Fe}: \mathrm{H}_{2} \mathrm{PO}_{2}: \mathrm{ClO}_{4}
$$

$$
1: 2.33: 0.55
$$

$\{1 \cdot 2.33: 0.66$

$\{1: 2.36: 0.65$

$1: 2.50: 0.60$

$1: 2.66: 0.55$

J : $2.83: 0.50$
Gefundene Prozente

$\begin{array}{ccc}\mathrm{Fe} & \mathrm{H}_{2} \mathrm{PO}_{2} & \mathrm{ClO}_{4} \\ 17.19 & 46.50 & 17.35 \\ 16.22 & 44.37 & 18.75 \\ 16.48 & 45.50 & 18.82 \\ 16.02 & 46.66 & 17.12 \\ 15.64 & 48.50 & 15.34 \\ 15.27 & 50.35 & 13.60\end{array}$

1) Whingand und Ensarabier, $Z$, anorg. Chem. 84 (1913), 367. 2) Sighe S. 23 u. 24. 
Diesen Prozenten liegen folgende Bestimmungen zugrunde:

$\begin{array}{lccccc} & \text { Substanz } & \mathrm{Fe}_{2} \mathrm{O}_{3} & \mathrm{Mg}_{2} \mathrm{P}_{2} \mathrm{O}_{7} & \text { Substanz } & \text { AgCl } \\ & \mathrm{g} & \mathrm{g} & \mathrm{g} & \mathrm{g} & \mathrm{g} \\ \text { a) } & \mathbf{0 . 3 6 7 1} & \mathbf{0 . 0 9 0 2} & 0.2922 & 0.5095 & 0.1274 \\ \text { b) } & 0.2984 & \mathbf{0 . 0 6 9 2} & 0.2266 & 0.2616 & \mathbf{0 . 0 7 0 7} \\ \text { c) } & 0.3030 & \mathbf{0 . 0 7 1 4} & 0.2360 & \mathbf{0 . 3 2 4 6} & \mathbf{0 . 0 8 8 0} \\ \text { d) } & 0.3205 & \mathbf{0 . 0 7 3 4} & 0.2560 & \mathbf{0 . 3 5 0 2} & 0.0864 \\ \text { e) } & \mathbf{0 . 2 8 4 4} & \mathbf{0 . 0 6 3 6} & 0.2361 & \mathbf{0 . 3 2 2 6} & 0.0713 \\ \text { f) } & \mathbf{0 . 3 0 7 8} & \mathbf{0 . 0 6 7 2} & 0.2653 & 0.4768 & 0.0934\end{array}$

\section{Reihe.}

Man fügt zu $5 \mathrm{ccm} 25 \%$ iger unterphosphoriger Säure steigende Mengen molekular-normaler Ferriperchloratlösung. Hierbei treten sogleich Fällungen von Perchlorat-hypophosphiten der Base ein. Diese sind alle arm an Uberchlorsäure und basischer als die Salze der vorigen Rrihe. Die Verhältnisse der äuBaren Glieder der Reihe sind $3 \mathrm{Fe}: 8 \mathrm{H}_{2} \mathrm{PO}_{2}: 1 \mathrm{ClO}_{4}$ und $3 \mathrm{Fe}: 7 \mathrm{H}_{2} \mathrm{PO}_{2}: 2(\mathrm{OH})$, die selbst jedoch nur annäherad erreicht werden. Am Ende hat man so ein fast reines basisches Ferrihypophosphit. Die Salze bilden weiße Pulver, die nicht wie die der anderen Reihe verklebt sind. Sie sind viel weniger explosiv, da sie ärmer an therchlorsäure sind; sie verpuffen nur beim Erhitzen, und zwar, je nach ihrem Gehalt an Uberchlorsäure, mehr oder weniger stark.

Angewandte Menge Zusammensetzung d $\leadsto$ Gefundene Prozente Ferriperchloratlösung erhaltenen Salzes

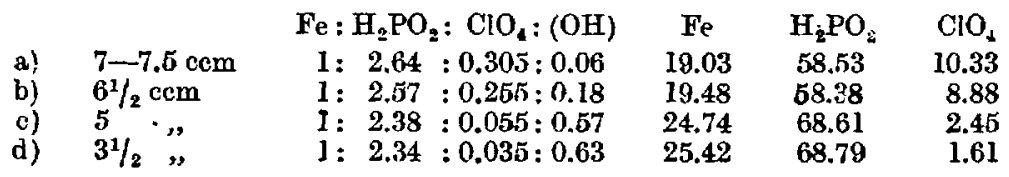

Diesen Angaben liegen folgende Werte zugrunde:

$\begin{array}{lccccc} & \text { Substanz } & \mathrm{Fe}_{2} \mathrm{O}_{3} & \mathrm{Mg}_{2} \mathrm{P}_{2} \mathrm{O}_{7} & \text { Substanz } & \mathrm{AgCl} \\ & \mathrm{g} & \mathrm{g} & \mathrm{g} & \mathrm{g} & \mathrm{g} \\ \mathrm{a}) & \mathbf{0 . 3 3 1 2} & 0.0901 & \mathbf{0 . 3 3 1 8} & 0.3024 & 0.0450 \\ \text { b) } & 0.2980 & 0.0830 & 0.2978 & 0.2986 & 0.0392 \\ \text { c) } & 0.3234 & \mathbf{0 . 1 1 4 4} & 0.3798 & \mathbf{0 . 4 5 5 3} & 0.0161 \\ \text { d) } & 0.1527 & \mathbf{0 . 0 5 5 5} & 0.1798 & \mathbf{0 . 4 4 8 4} & 0.0104\end{array}$

16. Sulfath ypophosphite.

Von den Reihen, die wir aus Ferrisulfat und unterphosphoriger Säure darstellten, führen wir im folgenden nur diejenige an, welche man durch Zusatz von unterphosphoriger Säure zu Ferrisulfatlösung bekommt. Die Ferrisulfatlösung stellten wir $1 / 2$ molekular-normal dar, indem wir $254 \mathrm{~g}$ $\mathrm{Fe}_{2}\left(\mathrm{SO}_{4}\right)_{3} \cdot 6 \mathrm{H}_{2} \mathrm{O}$ in 11 . Wasser lösten. Die unterphosphorige Säure war $25 \%$ ig.

Zu $20 \mathrm{ccm}$ der Ferrisulfatlösung fügt man, zuચächst kalt, $6 \mathrm{cem}$ der unterphosphorigen Sãure hinzu. Der hierbei zuerst entstehende Niederschlag löst sich beim Umrühren leicht wiєder auf. Die Lösung ist eigen- 
tümlich blaßrot. ${ }^{1}$ ) Fügt man zu der Lösung, während man sie mäBig erhitzt, tropfenweise die unterphosphorige Säure, im ganzen etwa $1-2 \mathrm{ccm}$, hinzu, so erhält man Ausscheidungen von Sulfathypophosphiten innerhalb nicht allzuweiter Grenzen schwankender Zusammensetzung. Die Verhältnisse liegen zwischen den Extremen

$$
3 \mathrm{Fe}: 6 \mathrm{H}_{2} \mathrm{PO}_{2}: 1 \mathrm{SO}_{4}: 1 \mathrm{OH} \text { und } 6 \mathrm{Fe}: 17 \mathrm{H}_{2} \mathrm{PO}_{2}: 1 \mathrm{SO} \text {. }
$$

Threm Habitus nach gleichen diese Verbindungen denen der ersten Reihe der Perchlorate Nr. 15 (S. 36).

Wir geben folgende Tabelle der Verbindungen, welche man auf die angegebene Weise erhält.

\begin{tabular}{ccccr} 
& $\begin{array}{c}\text { Zusammensetzung des } \\
\text { erhaltenen Salzes }\end{array}$ & \multicolumn{3}{c}{ Gefundene Prozente } \\
& $\mathrm{Fe}: \mathrm{H}_{2} \mathrm{PO}_{2}: \mathrm{SO}_{4}:(\mathrm{OH})$ & $\mathrm{Fe}$ & $\mathrm{H}_{2} \mathrm{PO}_{2}$ & $\mathrm{SO}_{4}$ \\
a) & $1: 2.04: 0.32: 0.32$ & $16.04(16.10)$ & 38.16 & 8.59 \\
b) & $1: 2.05: 0.33: 0.29$ & $20.36(18.85)$ & 48.62 & 10.26 \\
c) & $1: 2.26: 0.27: 0.20$ & $18.63(16.68)$ & 49.00 & 7.70 \\
d) & $1: 2.43: 0.25: 0.07$ & $19.38(18.53)$ & 64.95 & 8.04 \\
c) & $1: 2.66: 0.20-$ & $19.07(18.87)$ & 59.08 & 6.49 \\
f) & $1: 2.80: 0.17-$ & $18.95(18.81)$ & 61.83 & 6.51
\end{tabular}

Dieisen Angaben liegen folgende Bestimmungen zugrunde:

$\begin{array}{lcccccc} & \text { Substanz } & \mathrm{Fe}_{2} \mathrm{O}_{3} & \mathrm{Mg}_{2} \mathrm{P}_{2} \mathrm{O}_{7} & \text { Substanz } & \left(\mathrm{Fe}_{2} \mathrm{O}_{3}\right) & \mathrm{BaSO} \\ & \mathrm{g} & \mathrm{g} & \mathrm{g} & \mathrm{g} & \mathrm{g} & \mathrm{g} \\ \text { a) } & \mathbf{0 . 3 8 2 6} & 0.0648 & 0.1846 & 0.1902 & (0.0442) & 0.0404 \\ \text { b) } & 0.5374 & 0.1564 & 0.4472 & 0.5530 & (0.1490) & 0.1396 \\ \text { c) } & 0.3510 & 0.0935 & 0.2944 & 0.5149 & (0.1228) & 00963 \\ \text { d) } & 0.2996 & 0.0830 & 0.2811 & 0.3087 & (0.0818) & 0.0603 \\ \text { e) } & 0.3210 & 0.0875 & 0.3246 & 0.3006 & (0.0811) & 0.0474 \\ \text { f) } & \mathbf{0 . 2 6 7 2} & \mathbf{0 . 0 7 2 4} & 0.2828 & \mathbf{0 . 3 0 2 3} & (0.0813) & 0.0405\end{array}$

17. Chloridh ypophosphite.

Die Ferrichloridlösung war molekular-normal $\left(270.3 \mathrm{~g} \mathrm{FeCl}_{3}, 6 \mathrm{H}_{2} \mathrm{O}\right.$ in 11 Wasser), die unterphosphorige Säure $25 \%$ ig. Wir fügten $z u 10 \mathrm{ccm}$ der Ferrichloridlösung im ganzen etwa $8 \mathrm{ccm}$ der Säure hinzu, wobei man, wie bei cler Darstellung der Sulfate Nr. 16, diese am Ende tropfenweise und unter Erwärmen zusetzt. Die sich ausscheidenden weißlichen Salze stimmen in ihren Eigenschaften mit den vorigen überein. Die Mutterlauge ist auffallend kanariengelb. Die Verhältnisse der beiden äuBeren Glieder sind $3 \mathrm{Fe}: 7 \mathrm{H}_{2} \mathrm{PO}_{2}: 2 \mathrm{Cl}$ und $3 \mathrm{Fe}: 8 \mathrm{H}_{2} \mathrm{PO}_{2}: 1 \mathrm{OH}$. Man erhält somit messt schwach basische Salze, am Ende der Reihe steht sogar ein reines Ferrihypophosphit.

1) Die von uns beniutzte MERKsohe unterphosphorige Säure war calciumbaltig. Daher schied sich bei diesem Versuch auch Calciumsulfat aus, ron dem abfiltriert werden mußte. 
Folgende Tabelle gibt Aufschluß über die Reihe dieser Verbindungen:

Zusammensetzung des erhaltenen Salzes

$$
\mathrm{Fe}: \mathrm{H}_{2} \mathrm{PO}_{2}: \mathrm{Cl}:(\mathrm{OH})
$$

a)

b)

c)

d)

$$
\text { I: } 2.34: 0.65-
$$

$1: 2.43: 0.46: 0.11$

$1: 2.56: 0.20: 0.24$

$1: 2.65: 0.02: 0.33$
Gefundene Prozente

$\begin{array}{crr}\mathrm{Fe} & \mathrm{H}_{2} \mathrm{PO}_{2} & \mathrm{Cl} \\ 14.83 & 40.45 & 6.13 \\ 15.16 & 42.91 & 4.43 \\ 21.02 & 62.76 & 2.67 \\ 23.07 & 71.27 & 0.29\end{array}$

Diese Werte ergeben sich aus folgenden Bestimmungen:

$\begin{array}{cccccc} & \mathrm{g} & \mathrm{g} & \mathrm{g} & \mathrm{g} & \mathrm{g} \\ \text { a) } & 0.3325 & 0.0705 & 0.2302 & 0.2293 & 0.0568 \\ \text { b) } & 0.3272 & 0.0709 & 0.2403 & 0.3110 & 0.0557 \\ \text { c) } & \mathbf{0 . 2 5 1 8} & 0.0601 & 0.2705 & 0.3012 & 0.0325 \\ \text { d) } & \mathbf{0 . 2 3 4 7} & \mathbf{0 . 0 7 7 4} & 0.2863 & \mathbf{0 . 4 4 5 3} & 0.0053\end{array}$

\section{Ferrihypophosphite.}

(Vgl, oben S. 20ff.)

18. Dihypophosphit (3:8).

Das oben (S. 26) als Ausgangshypophosphit bezeichnete Salz stellt im ganzen das in Rede stehende Hypophosphit mit dem Verhältnis $3 \mathrm{Fe}: 8 \mathrm{H}_{2} \mathrm{PO}_{2}$ dar. Um es ganz rein darzustellen, fügt man zu einer Lösung von $10.8 \mathrm{~g} \mathrm{FeCl}_{3} .6 \mathrm{H}_{2} \mathrm{O}(4 / 100 \mathrm{Mole})$ in $40-50 \mathrm{ccm}$ Wasser eine solche von $12.7 \mathrm{~g} \mathrm{NaH}_{2} \mathrm{PO}_{2} . \mathrm{H}_{2} \mathrm{O}\left({ }^{12} /{ }_{100}\right.$ Mole) in $25-30 \mathrm{ccm}$ Wasser. ${ }^{1)}$ Man erwärmt dann mäbig, worauf sich der gebildete Niederschlag in ein gut absitzendes, sandiges Pulver verwandelt. (Arbeitet man in anderer Konzentration oder mit mehr oder weniger warmen Lösungen, so kann es vorkommen, daß das Salz mehr oder weniger basisch als das Salz 3:8 ausfällt. Arbeitet man verdünnter und heiB, so erhält man basischere Präparate, die zwischen 3:7 und 3:8 liegen, s. auch Nr. 19). Absaugen und auf Ton trocknen. Weißes lockeres Pulver mit einem geringen Stich ins Rötliche. Das Saly ist wasserhaltig, verwittert aber sehr rasch schon an der Luft. Präparate, die gerade lufttrocken waren, enthielten rund 24 Moleküle Wasser. Uber Chlorcalcium wird das Salz ziemlich rasch wasserfrei.

$$
\left[\mathrm{Fe}_{3}\left(\mathrm{H}_{2} \mathrm{PO}_{2}\right)_{\mathrm{B}}\right]\left(\mathrm{H}_{2} \mathrm{PO}_{2}\right)_{2}+24 \mathrm{H}_{2} \mathrm{O}
$$

(1137.4; für wasserfreies Salz Mol.-Gew. 705.01).

1. Salz mit $24 \mathrm{H}_{2} \mathrm{O}$.

I. $0.3026 \mathrm{~g}$ Substanz: $0.0634 \mathrm{~g} \mathrm{Fe} \mathrm{O}_{3}, 0.2352 \mathrm{~g} \mathrm{Mg}_{2} \mathrm{P}_{2} \mathrm{O}_{7}$.

II. $0.4006 \mathrm{~g}, \quad 0.0857 \mathrm{~g} \mathrm{Fe}_{2} \mathrm{O}_{3}, 0.3168 \mathrm{~g} \mathrm{Mg}_{2} \mathrm{P}_{2} \mathrm{O}_{7}$.

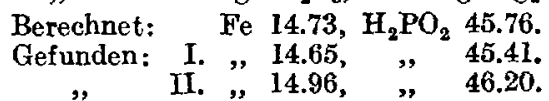

1) Man kann in diesem Falle auch ungekehrt die Eisenlösung zu der Natriumhypophosphitlösung fügen, wie bei der Darstellung des Ausgangshypophosphits (S. 26). 
2. Über $\mathrm{CaCl}_{2}$ entwässertes Salz.

I. $0.4193 \mathrm{~g}$ Substanz: $0.1410 \mathrm{~g} \mathrm{Fe}_{2} \mathrm{O}_{3}, 0.5272 \mathrm{~g} \mathrm{Mg}_{2} \mathrm{P}_{2} \mathrm{O}_{7}$.

II. $0.4873 \mathrm{~g} \quad, \quad 0.1653 \mathrm{~g} \mathrm{Fe}_{2} \mathrm{O}_{3}, 0.6158 \mathrm{~g} \mathrm{Mg} \mathrm{Mg}_{2} \mathrm{O}_{4}$.

$\begin{array}{crrrr}\text { Berechnet: } & \mathrm{Fe} & 23.76, & \mathrm{H}_{2} \mathrm{PO}_{2} & 73.83 . \\ \text { Gefunden: } & \text { I. } & 23.52, & \text {, } & \mathbf{7 3 . 4 6 .} \\ \text {," } & \text { II. }, & \mathbf{2 3 . 7 3 ,}, " & \mathbf{7 3 . 8 4} .\end{array}$

19. Sesquih ypophosphit $(6: 15)$.

Für die Darstellung dieses Salzes bemerken wir zuerst, dab frisch gefällte Hypophosphite in Wasser merklich löslich sind. Die Lösungen sind vollständig farblos; daß Ferrih ypophosphit gelöst ist, erkennt man an der Fällung von Ferribydroxyd auf Zusatz von Ammoniak.

Man löst $3.2 \mathrm{~g} \mathrm{(} 3 / 100$ Mole) $\mathrm{NaH}_{2} \mathrm{PO}_{2} . \mathrm{H}_{2} \mathrm{O}$ in $40 \mathrm{ccm}$ Wasser und fügt eine Lösung von $2.7 \mathrm{~g}(1 / 100 \mathrm{Mol}) \mathrm{FeCl}_{3} \cdot 6 \mathrm{H}_{2} \mathrm{O}$ in $60 \mathrm{ccm}$ Wasser hinzu. Bei dieser Konzentration der Lösungen tritt meistens kein Niederschlag auf oder wenn dies geschieht, läßt er sich durch Zusatz von Wasser wieder in Lösung bringen. Er stellt bereits das Sesquihypophosphit vor. Schönere Präparate und in besserer Ausbeute erbält man aber aus der obigen Lösung entweder durch Erhitzen oder indem man sie über Schwefelsäure im Vakuum verdunsten läßt. Der durch Erhitzen apgeschiedene Körper bildet eir voluminöses, feines, rein blaßrötliches Pulver. Das auskristallisierte Salz bildet eine sehr dünne, blaßrote Kruste. Unter dem Mikroskop beobachtet man mitunter bei guter Vergrößerung Blättchen von quadratischem oder rhombischem UmriB. Die Salze sind wasserhaltig, verlieren das Wasser aber so rasch, daß eine genauere Feststellung des Wassergehaltes nicht möglich ist.

$$
\left[\mathrm{Fe}_{3} \underset{\mathrm{OH}}{\left.\mathrm{H}_{2} \mathrm{PO}_{2}\right)_{6}}\right]\left(\mathrm{H}_{2} \mathrm{PO}_{2}\right)_{2}+\left[\mathrm{Fe}_{3} \underset{(\mathrm{OH})_{2}}{\left(\mathrm{H}_{2} \mathrm{PO}_{6}\right)_{7}}\right] \mathrm{H}_{2} \mathrm{PO}_{2}+\mathrm{xH}_{2} \mathrm{O}
$$

(Mol.-Gew für wassertreies Salz 1361.97).

1. Wasserhaltige Salze.

I. $0.3342 \mathrm{~g}$ Substanz: $0.0630 \mathrm{~g} \mathrm{Fe}_{2} \mathrm{O}_{3}=13.18 \%$ Fe und $0.2175 \mathrm{~g} \mathrm{Mg}_{2} \mathrm{P}_{2} \mathrm{O}_{7}$ $=38.02 \% \mathrm{H}_{2} \mathrm{PO}_{2}$.

II. $0.1995 \mathrm{~g}$ Substanz: $0.0430 \mathrm{~g} \mathrm{Fe} \mathrm{O}_{2} \mathrm{O}_{3}=15.07 \% \mathrm{Fe}$ und $0.1537 \mathrm{~g} \mathrm{Mg}_{2} \mathrm{P}_{2} \mathrm{O}_{2}$ $=45.01 \% \mathrm{H}_{2} \mathrm{PO}_{2}$.

III. $0.2963 \mathrm{~g}$ Substanz: $0.0869 \mathrm{~g} \mathrm{Fe} \mathrm{O}_{3}=20.51 \% \mathrm{Fe}$ und $0.3028 \mathrm{~g} \mathrm{Mg}_{8} \mathrm{P}_{2} \mathrm{O}$, $=59.71 \% \mathrm{H}_{\mathbf{2}} \mathrm{PO}_{2}$.

IV. $0.1294 \mathrm{~g}$ Substanz: $0.0422 \mathrm{~g} \mathrm{Fe}_{2} \mathrm{O}_{3}=22.81 \% \mathrm{Fe}$ und $0.1467 \mathrm{~g} \mathrm{Mg}_{2} \mathrm{P}_{2} \mathrm{O}_{7}$ $=66.23 \% \mathrm{H}_{2} \mathrm{PO}_{2}$.

Hiemach verhalten sich: $\mathrm{Fe}: \mathrm{H}_{2} \mathrm{PO}_{2}$

I. $1: 2.48$

II. $1: 2.56$

III. $1: 2.50$

IV. $1: 2.49$. 
2. Wasserfreie Salze.

I. $0.3026 \mathrm{~g}$ Substanz: $0.1063 \mathrm{~g} \mathrm{Fe}_{2} \mathrm{O}_{3}, 0.3721 \mathrm{~g} \mathrm{Mg} \mathrm{P}_{2} \mathrm{O}_{7}$.

II. $0.2890 \mathrm{~g} \quad, \quad 0.1020 \mathrm{~g} \mathrm{Fe} \mathrm{O}_{5}, 0.3542 \mathrm{~g} \mathrm{Mg} \mathrm{Mg}_{2} \mathrm{O}_{7}$.

Berechnet: $\mathrm{Fe} 24.60, \mathrm{H}_{2} \mathrm{PO}_{2} 70.02$.

Gefunden: I. , 24.57, , 71.84 .

" II. ", 24.68, " 71.60 .

20. Normales Hypophosphit $(3: 9) .{ }^{1}$ )

Zur Darstellung dieser Verbindung muß man ziemlich konzentrierte Lösungen benützen und die Eisenchloridlösung in der Hitze in diejenige des Natriumhypophosphits eingießen.

1. Man kann dieselben Lösungen benützen wie bei der Darstellung des Dihypophosphits (Nr. 18, S. 40), aber man muB die heiBe Eisenlösung ziemlich langsam in die gleichfalls erhitzte Natriumhypophosphitlösung eingießen, wobei die zuerst entstandene Fällung sich löst. Meistens genügt schon ein größerer Teil der Eisenlösung. Hierbei scheidet sich die Verbindung häufig in Form eines schweren, knirschenden, weißen Pulvers aus.

2. Man kann auch konzentriert arbeiten, indem man zu einer heißen Lösung von 5-10 g Natriumhypophosphit in gleichen Teilen Wasser von einer Ferrichloridlösung derselben Konzentration tropfenweise solange zusetzt, als der zuerst immer entstehende Niederschlag sich löst. Mit einem Male beginnt die Abscheidung einer reichlicheren Menge ues Hypophosphits. Die hier zuerst ausfallenden Niederschläge können manchmal schwach basisch sein (z. B. $3 \mathrm{Fe}: 8.7 \mathrm{H}_{2} \mathrm{PO}_{2}$ ). Aber die im Filtrat von diesen durch weiteren Zusatz von Eisenchlorid auf dieselbe Weise zu erhaltenen Hypophosphite sind normal. Dieses Präparat ist sehr feinpulvrig und blaßrosa.

3. Endlich bekommt man das normale Salz, jedoch nicht so leicht, bei der Behandlung von Ferrihydroxyd mit wäBriger unterphosphoriger Säure, wie im allgemeinen Teil erwähnt wurde (S. 15). Man muB frisch und kalt gefälltes Ferrihydroxyd benützen, da älteres oder heiß gefälltes nur sehr langsam oder unvollständig in das Hypophosphit verwandelt wird. Man verwendet auf 1 Molekül Ferrihydroxyd etwa 4-5 Moleküle unterphosphorige Säure; je mehr von dieser vorhanden ist, desto rascher verläuft die Umwandlung. Hierbei löst sich ein erheblicher Teil des Ferrihydroxyds und aus dieser Lösung kristallisiert das in Rede stehende Ferrihypophosphit besonders schön und in reichlicher Menge aus.

Auch durch längeres Digerieren von beliebigen Ferrih ypophosphiten mit unterphosphoriger Säure bekommt man das normale Salz, hat aber hierbei keine Gewähr für die Vollständigkeit der Umwandlung (s. S. 21).

1) Das Marksche Präparat stellt dieses Hypophosphit vor. 
Die so dargestellten normalen Hypophosphite sind meist wasserfrei. Nur wenn man (nach der zweiten Darstellungsweise) bei größerer Verdünnung, auch bei geringerer Hitze, ein Neutralsalz erhält, so ist es meist wie die anderen Hypophosphite wasserhaltig, ebenso wie auch das durch Digestion von Ferrihypophosphiten mit unterphosphoriger Säure erhaltene Präparat.

$$
\left[\mathrm{Fe}_{3}\left(\mathrm{H}_{2} \mathrm{PO}_{2}\right)_{6}\right]\left(\mathrm{H}_{2} \mathrm{PO}_{2}\right)_{3}(753.06) \text {. }
$$

I. $0.2165 \mathrm{~g}$ Substanz: $0.0683 \mathrm{~g} \mathrm{Fe}_{2} \mathrm{O}_{3}, 0.2870 \mathrm{~g} \mathrm{Mg}_{2} \mathrm{P}_{2} \mathrm{O}_{7}$.

II. $0.3032 \mathrm{~g} \quad, \quad 0.0966 \mathrm{~g} \mathrm{Fe} \mathrm{O}_{3}, 0.4032 \mathrm{~g} \mathrm{Mg}_{2} \mathrm{P}_{2} \mathrm{O}_{3}$.

III. $0.1950 \mathrm{~g} \quad, \quad 0.0620 \mathrm{~g} \mathrm{Fe}_{2} \mathrm{O}_{3}, 0.2624 \mathrm{~g} \mathrm{Mg}_{2} \mathrm{P}_{8} \mathrm{O}_{7}$.

Berechnet: $\quad \mathrm{Fo} 22,25$. $\mathrm{H}_{\mathbf{8}} \mathrm{PO}_{\mathbf{8}} 77.75$.

Gefunden: I. , 22.06, , 77.45 .

" II. "22.28, " 77.69 .

" III. ," 22,22, , 78,59 .

Wasserhaltige Salze.

I. $0.1661 \mathrm{~g}$ Substanz: $0.0449 \mathrm{~g} \mathrm{Fe}_{2} \mathrm{O}_{3}=18.91 \% \mathrm{Fe}$ und $0.1868 \mathrm{~g} \mathrm{Mg}_{2} \mathrm{P}_{2} \mathrm{O}_{\text {n }}$ $=65.70 \% \mathrm{H}_{2} \mathrm{PO}_{2}$.

II. $0.2173 \mathrm{~g}$ Substanz: $0.0501 \mathrm{~g} \mathrm{Fe}_{2} \mathrm{O}_{3}=16.13 \%$ Fe und $0.1710 \mathrm{~g} \mathrm{Mg}_{2} \mathrm{P}_{3} \mathrm{O}_{7}$ $=45.97 \% \mathrm{H}_{2} \mathrm{PO}_{2}$.

Hiernach verhalten sich: $\mathrm{Fe}: \mathrm{H}_{2} \mathrm{PO}_{2}$

I. 1 : 2.98 .

II. 1 : 3.06 .

21. Monohypophosphit $(3: 7)$.

Zur Darstellung dieser Verbindung kocht man ein Ferrihypophosphit, am einfachsten das Ausgangshypophosphit 3:8, aber auch ein beliebiges anderes Salz der Base, mit Wasser (5 g mit etwa $50 \mathrm{ccm}$ ) 2-3 Stunden am RückfluBkühler. Absaugen, mit heißem Wasser etwas nachwaschen, an der Luft trocknen. Im Filtrat ist zweiwertiges Eisen und Phosphorsäure nachweisbar (S. 21).

Es kommt zuweilen vor, daB nebenher etwas Ferrihydroxyd entsteht und das Präparat verupreinigt. Reine Praparate sind völlig weiß, mit einem geringen Stich ins Rötliche; sie bestehen aus eigentümlich kugligen, lockeren Gebilden. Das frische wasserhaltige Salz verwittert rasch an der Luft, indem es sichtbar zerstäubt. Die Feststellung des Wassergehaltes ist daher nicht genau ausführbar. Bei lufttrockenem Salz kommen auf ein Atom Eisen etwa 12 Moleküle Wasser. ${ }^{1}$ )

$\left[\mathrm{Fe}_{3}\left(\mathrm{OH}_{2} \mathrm{PO}_{2}\right)_{6}\right] \mathrm{H}_{2} \mathrm{PO}_{2}+\mathrm{xH}_{2} \mathrm{O}$ (Mol.-Gew. für wasserfreies Salz 656.96).

1) Eine andere Bildungsweise ist im Folgenden (Nr. 22) beschrieben. 
1. Wasserhaltige Salze.

I. $0.4810 \mathrm{~g}$ Substanz: $0.0857 \mathrm{~g} \mathrm{Fe}_{2} \mathrm{O}_{3}=12.46 \%$ Fe und $0.2816 \mathrm{~g} \mathrm{Mg}_{2} \mathrm{P}_{2} \mathrm{O}_{7}$ $=34.20 \% \mathrm{H}_{2} \mathrm{PO}_{2}$.

II. $0.4272 \mathrm{~g}$ Substanz: $0.0804 \mathrm{~g} \mathrm{Fe}_{2} \mathrm{O}_{3}=13.16 \% \mathrm{Fe}$ und $0.2624 \mathrm{~g} \mathrm{Mg}_{2} \mathrm{P}_{2} \mathrm{O}_{7}$ $\bar{\sigma} 35.88 \% \mathrm{H}_{2} \mathrm{P}_{2} \mathrm{O}_{2}$.

III. $0.2589 \mathrm{~g}$ Substanz: $0.0513 \mathrm{~g} \mathrm{Fe}_{2} \mathrm{O}_{3}=13.86 \% \mathrm{Fe}$ und $0.1670 \mathrm{~g} \mathrm{Mg}_{2} \mathrm{P}_{2} \mathrm{O}_{7}$ $=37.68 \% \mathrm{H}_{2} \mathrm{PO}_{2}$.

IV. $0.4968 \mathrm{~g}$ Substanz: $0.1116 \mathrm{~g} \mathrm{Fe} \mathrm{O}_{\mathrm{a}}=15.71 \% \mathrm{Fe}$ und $0.3617 \mathrm{~g} \mathrm{Mg}_{2} \mathrm{P}_{2} \mathrm{O}_{7}$ $=42.54 \% \quad \mathrm{H}_{2} \mathrm{PO}_{2}$.

V. $0.1431 \mathrm{~g}$ Substanz: $0.0500 \mathrm{~g} \mathrm{Fe}_{2} \mathrm{O}_{3}=24.44 \%$ Fe und $0.1648 \mathrm{~g} \mathrm{Mg}_{2} \mathrm{P}_{2} \mathrm{O}_{7}$ $=67.30 \% \mathrm{H}_{2} \mathrm{PO}_{2}$.

Hiernach verbalten sich: $\mathrm{Fe}: \mathrm{H}_{2} \mathrm{PO}_{3}$

$$
\begin{aligned}
\text { I. } & 1: 2.36 \\
\text { II. } & 1: 2.34 \\
\text { III. } & 1: 2.33 \\
\text { IV. } & 1: 2.32 \\
\text { V. } & 1: 2.36 .
\end{aligned}
$$

2. Wasserfreie Salze.

II. $0.3089 \mathrm{~g}$ Substanz: $0.1124 \mathrm{~g} \mathrm{Fe}_{2} \mathrm{O}_{3}, 0.3702 \mathrm{~g} \mathrm{Mg}_{2} \mathrm{P}_{2} \mathrm{O}_{7}$.

II. $0.3504 \mathrm{~g} \quad " \quad 0.1273 \mathrm{~g} \mathrm{Fe} \mathrm{O}_{3}, 0.4155 \mathrm{~g} \mathrm{Mg} 2 \mathrm{P}_{2} \mathrm{O}_{7}$.

Berechnet: $\quad \mathrm{Fe} 25.50, \mathrm{H}_{2} \mathrm{PO}_{2} 69.32$.

Gefunden: I. , 25.45, , 70.02 .

", II. ", $25.41, \quad, \quad 69.28$.

22. Hypophosphit der Pentahypophosphito-triferri-Base $(3: 6)$.

Man behandelt ein ganz beliebiges \$alz der Base längere Zeit (2 bis 4 Tage) mit kaltem Wasser. Bei einem gelbgefärbten Salze, wie dem oktaedrischen Nr. 8, kann man den UmwandlungsprozeB genau verfolgen, da das entstehende Salz weiB ist. Es fördert die Umwandlung, wenn man, besonders am Anfang, das Wasser zuweilen durch neues ersetzt. Aus den abgegossenen wäßrigen Lösungen scheidet sich beim Verdunsten im Vakuum über Schwefelsäure das vorhergehende Salz (Nr.21) in feinen rötlichen Krusten aus. Der zu hohe Gehalt an unterphosphoriger Säure, den man bei manchen Präparaten findet, rührt daher, daß beim Verdunsten der überstehenden Flüssigkeit sich von dem letzteren Salz beimengt.

Dieses Salz erhält man merkwürdigerweise auch, indem ein beliebiges Hypophosphit, auch ein basisches Ferrihypophosphit, mit unterphosphoriger Säure digeriert, z. B. $3 \mathrm{~g}$ des Ausgangshypophosphits mit

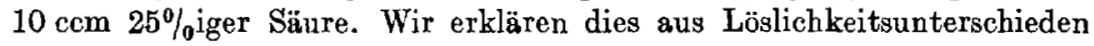
des Hypophosphits der Pentabase gegenüber den Hypophosphiten der Hexabase (S. 21).

$\left[\mathrm{Fe}_{3} \underset{\left.(\mathrm{OH})_{3}\right)_{5}}{\left(\mathrm{H}_{2} \mathrm{PO}_{2}\right.} \mathrm{H}_{2} \mathrm{PO}_{2}+\mathrm{xH}_{2} \mathrm{O}\right.$ (Mol.-Gew. für wasserfreies Salz 608.91). 
1. Wasserhaltige Salze.

I. $0.4196 \mathrm{~g}$ Substanz: $0.0863 \mathrm{~g} \mathrm{Fe}_{2} \mathrm{O}_{3}=14.38 \%$ Fe und $0.2764 \mathrm{~g} \mathrm{Mg}_{2} \mathrm{P}_{2} \mathrm{O}_{2}$ $=33.52 \% \mathrm{H}_{2} \mathrm{PO}_{2}$.

II. $0.5955 \mathrm{~g}$ Substanz: $0.1345 \mathrm{~g} \mathrm{Fe}_{2} \mathrm{O}_{3}=15.80 \% \mathrm{Fe}$ und $0.3677 \mathrm{~g} \mathrm{Mg}_{2} \mathrm{P}_{2} \mathrm{O}_{7}$ $=36.07 \% \mathrm{H}_{2} \mathrm{PO}_{2}$.

III. $0.3840 \mathrm{~g}$ Substanz: $0.1199 \mathrm{~g} \mathrm{Fe}_{2} \mathrm{O}_{3}=21.84 \% \mathrm{Fe}$ und $0.2046 \mathrm{~g} \mathrm{Mg}_{2} \mathrm{P}_{2} \mathrm{O}_{7}$ $=49.34 \% \mathrm{H}_{2} \mathrm{PO}_{2}$.

IV. $0.4125 \mathrm{~g}$ Substanz: $0.1413 \mathrm{~g} \mathrm{Fe}_{2} \mathrm{O}_{3}=23.96 \%$ Fe und $0.4004 \mathrm{~g} \mathrm{Mg}_{2} \mathrm{P}_{8} \mathrm{O}_{7}$ $=56.71 \% \mathrm{H}_{2} \mathrm{PO}_{2}$.

Hiernach verhalten sich: $\quad \mathrm{Fe}: \mathrm{H}_{2} \mathrm{PO}_{2}$

$$
\begin{aligned}
\text { I. } & 1: 2.00 \\
\text { II. } & 1: 1.96 \\
\text { III. } & 1: 1.94 \\
\text { IV. } & 1: 2.03 .
\end{aligned}
$$

2. Wasserfreie Salze.

I. $0.3218 \mathrm{~g}$ Substanz: $0.1262 \mathrm{~g} \mathrm{Fe}_{2} \mathrm{O}_{3}, 0.3626 \mathrm{~g} \mathrm{Mg}_{2} \mathrm{P}_{2} \mathrm{O}_{2}$.

II. $0.2946 \mathrm{~g} \quad, \quad 0.1164 \mathrm{~g} \mathrm{Fe}_{2} \mathrm{O}_{3}, 0.3215 \mathrm{~g} \mathrm{Mg}_{2} \mathrm{P}_{2} \mathrm{O}_{7}$.

Berechnet: $\mathrm{Fe} 27.51, \mathrm{H}_{2} \mathrm{PO}_{2} 64.11$.

Gefunden: I. ,27.43, , 65.83 .

" II. " $27.63, \quad " 63.76$.

Tubingen, Chemisches Laboratorium der Universität, 18. Oktober 1918.

Bei der Redaktion eingegangen am 19. Oktober 1918. 